quatrième série-tome $46 \quad$ fascicule 1 janvier-février 2013

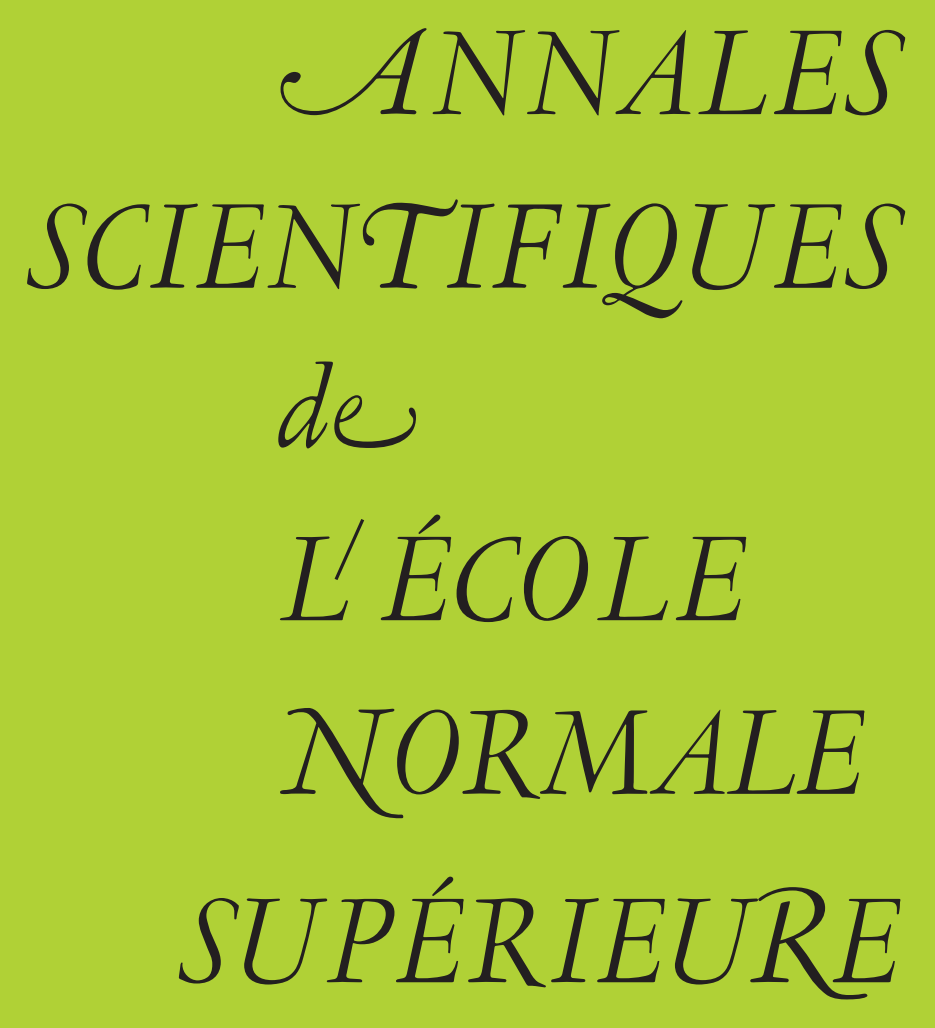

Sébastien GOUËZEL \& Steven P. LALLEY

Random Walks on Co-Compact Fuchsian Groups 


\title{
RANDOM WALKS ON CO-COMPACT FUCHSIAN GROUPS
}

\author{
By SÉbastien GOUËZEL and Steven P. LALLEY
}

\begin{abstract}
Aвstract. - It is proved that the Green's function of a symmetric finite range random walk on a co-compact Fuchsian group decays exponentially in distance at the radius of convergence $R$. It is also shown that Ancona's inequalities extend to $R$, and therefore that the Martin boundary for $R$-potentials coincides with the natural geometric boundary $S^{1}$, and that the Martin kernel is uniformly Hölder continuous. Finally, this implies a local limit theorem for the transition probabilities: in the aperiodic case, $p^{n}(x, y) \sim C_{x, y} R^{-n} n^{-3 / 2}$.
\end{abstract}

RÉSUMÉ. - Considérons une marche aléatoire symétrique à support fini sur un groupe fuchsien cocompact. Nous montrons que la fonction de Green à son rayon de convergence $R$ décroît exponentiellement vite en fonction de la distance à l'origine. Nous montrons également que les inégalités d'Ancona s'étendent jusqu'au paramètre $R$, et par conséquent que la frontière de Martin pour les $R$-potentiels s'identifie avec la frontière géométrique $S^{1}$. De plus, le noyau de Martin correspondant est höldérien. Ces résultats sont utilisés pour démontrer un théorème limite local pour les probabilités de transition : dans le cas apériodique, $p^{n}(x, y) \sim C_{x, y} R^{-n} n^{-3 / 2}$.

\section{Introduction}

\subsection{Green's function and Martin boundary}

A (right) random walk on a countable group $\Gamma$ is a discrete-time Markov chain $\left\{X_{n}\right\}_{n \geq 0}$ of the form

$$
X_{n}=x \xi_{1} \xi_{2} \cdots \xi_{n}
$$

where $\xi_{1}, \xi_{2}, \ldots$ are independent, identically distributed $\Gamma$-valued random variables. The distribution of $\xi_{i}$ is the step distribution of the random walk. The random walk is said to be symmetric if its step distribution is invariant under the mapping $x \mapsto x^{-1}$, and finite-range if the step distribution has finite support. The Green's function is the generating function of the

Second author supported by NSF grant DMS - 0805755. 
transition probabilities: for $x, y \in \Gamma$ and $0 \leq r<1$ it is defined by the absolutely convergent series

$$
G_{r}(x, y):=\sum_{n=0}^{\infty} P^{x}\left\{X_{n}=y\right\} r^{n}=G_{r}\left(1, x^{-1} y\right) ;
$$

here $P^{x}$ is the probability measure on path space governing the random walk with initial point $x$. If the random walk is irreducible (that is, if the semigroup generated by the support of the step distribution is $\Gamma$ ) then the radius of convergence $R$ of the series (1) is the same for all pairs $x, y$. Moreover, if the random walk is symmetric, then $1 / R$ is the spectral radius of the transition operator. By a fundamental theorem of Kesten [25], if the group $\Gamma$ is finitely generated and nonamenable then $R>1$. Moreover, in this case the Green's function is finite at its radius of convergence (cf. [39], ch. 2): for all $x, y \in \Gamma$,

$$
G_{R}(x, y)<\infty .
$$

The Green's function is of central importance in the study of random walks. Clearly, it encapsulates information about the transition probabilities; in Theorem 9.1, we show that the local asymptotic behavior of the transition probabilities can be deduced from the singular behavior of the Green's function at its radius of convergence. The Green's function is also the key to the potential theory associated with the random walk: in particular, it determines the Martin boundary for $r$-potential theory. A prominent theme in the study of random walks on nonabelian groups has been the relationship between the geometry of the group and the nature of the Martin boundary. A landmark result here is a theorem of Ancona [2] describing the Martin boundary for random walks with finitely supported step distributions on hyperbolic groups: Ancona proves that for every $r \in(0, R)$ the Martin boundary for $r$-potential theory coincides with the geometric (Gromov) boundary, in a sense made precise below. (Series [35] had earlier established this in the special case $r=1$ when the group is co-compact Fuchsian. See also [3] and [1] for related results concerning LaplaceBeltrami operators on Cartan manifolds.)

It is natural to ask whether Ancona's theorem extends to $r=R$, that is, if the Martin boundary is stable (see [33] for the terminology) through the entire range $(0, R]$. One of the main results of this paper (Theorem 1.3) provides an affirmative answer in the special case of symmetric, finite-range random walk on a co-compact Fuchsian group, i.e., a cocompact, discrete subgroup of $P S L(2, \mathbb{R})$. Any co-compact Fuchsian group acts as a discrete group of isometries of the hyperbolic disk, and so its Cayley graph can be embedded quasiisometrically in the hyperbolic disk; this implies that its Gromov boundary is the circle $S^{1}$ at infinity.

THeOREM 1.1. - For any symmetric, irreducible, finite-range random walk on a co-compact Fuchsian group $\Gamma$, the Martin boundary for $R$-potentials coincides with the geometric boundary $S^{1}=\partial \Gamma$. Moreover, all elements of the Martin boundary are minimal.

This assertion means that (a) for every geodesic ray $y_{0}, y_{1}, y_{2}, \ldots$ in the Cayley graph that converges to a point $\zeta \in \partial \Gamma$ and for every $x \in \Gamma$,

$$
\lim _{n \rightarrow \infty} \frac{G_{R}\left(x, y_{n}\right)}{G_{R}\left(1, y_{n}\right)}=K_{R}(x, \zeta)=K(x, \zeta)
$$

$4^{\mathrm{e}}$ SÉRIE - TOME $46-2013-\mathrm{N}^{\circ} 1$ 
exists; (b) for each $\zeta \in \partial \Gamma$ the function $K_{\zeta}(x):=K(x, \zeta)$ is a minimal, positive $R$-harmonic function of $x$; (c) for distinct points $\zeta, \zeta^{\prime} \in \partial \Gamma$ the functions $K_{\zeta}$ and $K_{\zeta^{\prime}}$ are different; and (d) the topology of pointwise convergence on $\left\{K_{\zeta}\right\}_{\zeta \in \partial \Gamma}$ coincides with the usual topology on $\partial \Gamma=S^{1}$.

Our results also yield explicit rates for the convergence (3), and imply that the Martin kernel $K_{r}(x, \zeta)$ is Hölder continuous in $\zeta$ relative to the usual Euclidean metric (or any visual metric — see [23] for the definition) on $S^{1}=\partial \Gamma$.

THEOREM 1.2. - For any symmetric, irreducible, finite-range random walk on a co-compact Fuchsian group $\Gamma$, there exists $\varrho<1$ such that for every $1 \leq r \leq R$ and every geodesic ray $1=y_{0}, y_{1}, y_{2}, \ldots$ converging to a point $\zeta \in \partial \Gamma$,

$$
\left|\frac{G_{r}\left(x, y_{n}\right)}{G_{r}\left(1, y_{n}\right)}-K_{r}(x, \zeta)\right| \leq C_{x} \varrho^{n} .
$$

The constants $C_{x}<\infty$ depend on $x \in \Gamma$ but not on $r \leq R$. Consequently, for each $x \in \Gamma$ and $r \leq R$ the function $\zeta \mapsto K_{r}(x, \zeta)$ is Hölder continuous in $\zeta$ relative to the Euclidean metric on $S^{1}=\partial \Gamma$, for some exponent not depending on $r \leq R$.

The exponential convergence (4) and the Hölder continuity of the Martin kernel for $r=1$ were established by Series [34] for random walks on Fuchsian groups. Similar results for the Laplace-Beltrami operator on negatively curved Cartan manifolds were proved by Anderson and Schoen [3]. The methods of [3] were adapted by Ledrappier [30] to prove that Series' results extend to all random walks on a free group, and Ledrappier's proof was extended by Izumi, Neshvaev, and Okayasu [22] to prove that for a random walk on a non-elementary hyperbolic group the Martin kernel $K_{1}(x, \zeta)$ is Hölder continuous in $\zeta$. All of these proofs rest on inequalities of the type discussed in Section 1.2 below. Theorem 1.3 below asserts (among other things) that similar estimates are valid for all $G_{r}$ uniformly for $r \leq R$. Given these, the proof of [22] applies almost verbatim to establish Theorem 1.2. We will give some additional details in Paragraph 4.2.

\subsection{Ancona's inequalities}

The crux of Ancona's argument in [2] was a system of inequalities that assert, roughly, that the Green's function $G_{r}(x, y)$ is nearly submultiplicative in the arguments $x, y \in \Gamma$. Ancona [2] proved that such inequalities always hold for $r<R$ : in particular, he proved, for a random walk with finitely supported step distribution on a hyperbolic group, that for each $r<R$ there is a constant $C_{r}<\infty$ such that for every geodesic segment $x_{0} x_{1} \cdots x_{m}$ in (the Cayley graph of) $\Gamma$,

$$
G_{r}\left(x_{0}, x_{m}\right) \leq C_{r} G_{r}\left(x_{0}, x_{k}\right) G_{r}\left(x_{k}, x_{m}\right) \quad \forall 1 \leq k \leq m .
$$

His argument depends in an essential way on the hypothesis $r<R$ (cf. his Condition (*)), and it leaves open the possibility that the constants $C_{r}$ in the inequality (5) might blow up as $r \rightarrow R$. For finite-range random walk on a free group it can be shown, by direct calculation, that the constants $C_{r}$ remain bounded as $r \rightarrow R$, and that the inequalities (5) remain valid at $r=R$ (cf. [27]). The following result asserts that the same is true for symmetric random walks on a co-compact Fuchsian group. 
THEOREM 1.3. - For any symmetric, irreducible, finite-range random walk on a co-compact Fuchsian group $\Gamma$,

(A) the Green's function $G_{R}(1, x)$ decays exponentially in $|x|:=d(1, x)$; and

(B) Ancona's inequalities (5) hold for all $r \leq R$, with a constant $C$ independent of $r$.

Note 1.4. - Here and throughout the paper $d(x, y)$ denotes the distance between the vertices $x$ and $y$ in the Cayley graph $G^{\Gamma}$, equivalently, distance in the word metric. Exponential decay of the Green's function means uniform exponential decay in all directions, that is, there are constants $C<\infty$ and $\varrho<1$ such that for all $x, y \in \Gamma$,

$$
G_{R}(x, y) \leq C \varrho^{d(x, y)} .
$$

A very simple argument (see Lemma 2.1 below) shows that for a symmetric random walk on any nonamenable group $G_{R}(1, x) \rightarrow 0$ as $|x| \rightarrow \infty$. Given this, it is routine to show that exponential decay of the Green's function follows from Ancona's inequalities. However, we will argue in the other direction, first providing an independent proof of exponential decay in Subsection 3.3, and then deducing Ancona's inequalities from it in Section 4.

Note 1.5. - Theorem 1.3 (A) is a discrete analogue of one of the main results (Theorem B) of Hamenstaedt [21] concerning the Green's function of the Laplacian on the universal cover of a compact negatively curved manifold. Unfortunately, Hamenstaedt's proof appears to have a serious error. ${ }^{(1)}$ The approach taken here bears no resemblance to that of [21].

Theorem 1.3 is proved in Sections 3 and 4 below. The argument uses the planarity of the Cayley graph in an essential way. It also relies on the simple estimate

$$
\lim _{|x| \rightarrow \infty} G_{R}(1, x)=0
$$

that we derive from the symmetry of the random walk. While this estimate is not true in general without the symmetry assumption, we nevertheless conjecture that Ancona's inequalities and the identification of the Martin boundary at $r=R$ hold in general.

\subsection{Decay at infinity of the Green's function}

Neither Ancona's result nor Theorem 1.3 gives any information about how the uniform exponential decay rate $\varrho$ depends on the step distribution of the random walk. In fact, the Green's function $G_{r}(1, x)$ decays at different rates in different directions $x \rightarrow \partial \Gamma$. To quantify the overall decay, consider the behavior of the Green's function over the entire sphere $S_{m}$ of radius $m$ centered at 1 in the Cayley graph $G^{\Gamma}$. If $\Gamma$ is a nonelementary Fuchsian group then the cardinality of the sphere $S_{m}$ grows exponentially in $m$ (see Corollary 5.5 in Section 5), that is, there exist constants $C>0$ and $\zeta>1$ such that as $m \rightarrow \infty$,

$$
\left|S_{m}\right| \sim C \zeta^{m} \text {. }
$$

\footnotetext{
(1) The error is in the proof of Lemma 3.1: The claim is made that a lower bound on a finite measure implies a lower bound for its Hausdorff-Billingsley dimension relative to another measure. This is false - in fact such a lower bound on measure implies an upper bound on its Hausdorff-Billingsley dimension.

4 e SÉRIE - TOME $46-2013-$ N $^{\circ} 1$
} 
THEOREM 1.6. - For any symmetric, irreducible, finite-range random walk on a co-compact Fuchsian group $\Gamma$,

$$
\lim _{m \rightarrow \infty} \sum_{x \in S_{m}} G_{R}(1, x)^{2}=C>0
$$

exists and is finite, and

$$
\#\left\{x \in \Gamma: G_{R}(1, x) \geq \varepsilon\right\} \asymp \varepsilon^{-2}
$$

as $\varepsilon \rightarrow 0$. (Here $\asymp$ means that the ratio of the two sides remains bounded away from 0 and $\infty$.)

The proof is carried out in Sections 6-7 below (cf. Propositions 6.2 and 7.1), using the fact that any hyperbolic group has an automatic structure [19]. The automatic structure will permit us to use the theory of Gibbs states and thermodynamic formalism of Bowen [11], ch. 1. Theorem 1.2 is essential for this, as the theory developed in [11] applies only to Hölder continuous functions.

It is likely that $\asymp$ can be replaced by $\sim$ in (8). There is a simple heuristic argument that suggests why the sums $\sum_{x \in S_{m}} G_{R}(1, x)^{2}$ should remain bounded as $m \rightarrow \infty$ : Since the random walk is $R$-transient, the contribution to $G_{R}(1,1)<\infty$ from random walk paths that visit $S_{m}$ and then return to 1 is bounded (by $G_{R}(1,1)$ ). For any $x \in S_{m}$, the term $G_{R}(1, x)^{2} / G_{R}(1,1)$ is the contribution to $G_{R}(1,1)$ from paths that visit $x$ before returning to 1 . Thus, if $G_{R}(1, x)$ is not substantially larger than

$$
\sum_{n=1}^{\infty} P^{1}\left\{X_{n}=x \text { and } \tau(m)=n\right\} R^{n},
$$

where $\tau(m)$ is the time of the first visit to $S_{m}$, then the sum in (7) should be of the same order of magnitude as the total contribution to $G_{R}(1,1)<\infty$ from random walk paths that visit $S_{m}$ and then return to 1 . Of course, the difficulty in making this heuristic argument rigorous is that a priori one does not know that paths that visit $x$ are likely to be making their first visits to $S_{m}$; it is Ancona's inequality (5) that ultimately fills the gap.

Note 1.7. - A simple argument shows that for $r>1$ the sum of the Green's function on the sphere $S_{m}$, unlike the sum of its square, explodes as $m \rightarrow \infty$. Fix $1<r \leq R$ and $m \geq 1$. Let $C_{0}$ bound the size of the jumps of the random walk, and let $\tilde{S}_{m}$ be the set of points with $d(1, x) \in\left[m, m+C_{0}\right)$. Since $X_{n}$ is transient, it will, with probability one, eventually visit the annulus $\tilde{S}_{m}$. The minimum number of steps needed to reach $\tilde{S}_{m}$ is at least $m / C_{0}$. Hence,

$$
\begin{aligned}
\sum_{x \in \tilde{S}_{m}} G_{r}(1, x) & =\sum_{n=m / C_{0}}^{\infty} \sum_{x \in \tilde{S}_{m}} P^{1}\left\{X_{n}=x\right\} r^{n} \\
& \geq r^{m / C_{0}} \sum_{n=m / C}^{\infty} P^{1}\left\{X_{n} \in \tilde{S}_{m}\right\} \\
& \geq r^{m / C_{0}} P^{1}\left\{X_{n} \in \tilde{S}_{m} \text { for some } n\right\} \\
& =r^{m / C_{0}} .
\end{aligned}
$$

Hence, $\sum_{x \in \tilde{S}_{m}} G_{r}(1, x)$ diverges. The divergence of $\sum_{x \in S_{m}} G_{r}(1, x)$ readily follows if the random walk is irreducible. 
Note 1.8. - There are some precedents for the result (7). Ledrappier [29] has shown that for Brownian motion on the universal cover of a compact Riemannian manifold of negative curvature, the integral of the Green's function $G_{1}(x, y)=\int_{0}^{\infty} p_{t}(x, y) d t$ over the sphere $S(\varrho, x)$ of radius $\varrho$ centered at a fixed point $x$ converges as $\varrho \rightarrow \infty$ to a positive constant $C$ independent of $x$. Hamenstaedt [21] proves in the same context that the integral of $G_{R}^{2}$ over $S(\varrho, x)$ remains bounded as the radius $\varrho \rightarrow \infty$. Our arguments (see Note 6.3 in Sec. 6) show that for finite range irreducible random walk on a co-compact Fuchsian group the following is true: for each value of $r$ there exists a power $1 \leq \theta=\theta(r) \leq 2$ such that

$$
\lim _{m \rightarrow \infty} \sum_{x \in S_{m}} G_{r}(1, x)^{\theta}=C_{r}>0 .
$$

\subsection{Critical exponent for the Green's function}

Theorem 1.6 implies that the behavior of the Green's function $G_{R}(x, y)$ at the radius of convergence as $y$ approaches the geometric boundary is intimately related to the behavior of $G_{r}(x, y)$ as $r \uparrow R$. The connection between the two is rooted in the following set of differential equations.

Proposition 1.9. - For any random walk on any discrete group, the Green's functions satisfy

$$
\frac{d}{d r} G_{r}(x, y)=r^{-1} \sum_{z \in \Gamma} G_{r}(x, z) G_{r}(z, y)-r^{-1} G_{r}(x, y) \quad \forall 0 \leq r<R .
$$

Although the proof is elementary (cf. Section 2.1 below) these differential equations have not (to our knowledge) been observed before. Theorem 1.6 implies that the sum in Equation (9) blows up as $r \rightarrow R$-; this is what causes the singularity of $r \mapsto G_{r}(1,1)$ at $r=R$. The rate at which the sum blows up determines the critical exponent for the Green's function, that is, the exponent $\alpha$ for which $G_{R}(1,1)-G_{r}(1,1) \sim C(R-r)^{\alpha}$. The following theorem asserts that the critical exponent is $1 / 2$.

THeOREm 1.10. - For any symmetric, irreducible, finite-range random walk on a cocompact Fuchsian group $\Gamma$, there exist constants $C_{x, y}>0$ such that as $r \rightarrow R-$,

$$
\begin{aligned}
G_{R}(x, y)-G_{r}(x, y) & \sim C_{x, y} \sqrt{R-r} \text { and } \\
d G_{r}(x, y) / d r & \sim \frac{1}{2} C_{x, y} / \sqrt{R-r} .
\end{aligned}
$$

The proof of Theorem 1.10 is given in Section 8. Like the proof of Theorem 1.6, it uses the existence of an automatic structure and the attendant thermodynamic formalism. It also relies critically on the conclusion of Theorem 1.6, which determines the value of the key thermodynamic variable.

The behavior of the generating function $G_{r}(1,1)$ in the neighborhood of the singularity $r=R$ is of interest because it reflects the asymptotic behavior of the coefficients $P^{1}\left\{X_{n}=1\right\}$ as $n \rightarrow \infty$. In Section 9 we will show that Theorem 1.10, in conjunction with Karamata's Tauberian Theorem, implies the following local limit theorem. 
THEOREM 1.11. - For any symmetric, irreducible, finite-range, aperiodic random walk on a co-compact Fuchsian group with spectral radius $R^{-1}$, there exist constants $C_{x, y}>0$ such that for all $x, y \in \Gamma$,

$$
p^{n}(x, y) \sim C_{x, y} R^{-n} n^{-3 / 2} .
$$

If the random walk is not aperiodic, these asymptotics hold for even (resp. odd) $n$ if the distance from $x$ to $y$ is even (resp. odd).

According to a theorem of Bougerol [10], the transition probability densities of a random walk on a semi-simple Lie group follow a similar asymptotic law, provided the step distribution is rapidly decaying and has an absolutely continuous component with respect to the Haar measure. The exponential decay rate depends on the step distribution, but the critical exponent (the power of $n$ in the asymptotic formula, in our case $3 / 2$ ) depends only on the rank and the number of positive, indivisible roots of the group. Theorem 1.11 shows that - at least for $S L(2, \mathbb{R})$--the critical exponent is inherited by a large class of co-compact discrete subgroups. For random walks on free groups [18], [27], most free products [38], and virtually free groups that are not virtually cyclic (including $S L_{2}(\mathbb{Z})$ ), [37], [28], [31], [40] local limit theorems of the form (12) have been known for some time. In all of these cases the Green's functions are algebraic functions of $r$. We expect (but cannot prove) that for symmetric, finiterange random walks on co-compact Fuchsian groups the Green's functions are not algebraic. However, we will prove in Section 9 (Theorem 9.3) that the Green's function admits an analytic continuation to a doubly slit plane $\mathbb{C} \backslash([R, \infty) \cup(-\infty,-R(1+\varepsilon)])$, and Theorem 1.10 implies that if the singularity at $r=R$ is a branch point then it must be of order 2 . If it could be shown that the singularity is indeed a branch point then it would follow that the transition probabilities have complete asymptotic expansions in powers of $n^{-1 / 2}$.

The most important step in our program - the proof of Ancona's inequalities (Theorem 1.3) - depends heavily on the planarity of the Cayley graph of the group. The derivation of the subsequent results (including Theorems 1.6, 1.10 and 1.11) uses thermodynamic formalism, which is possible since the Markov automaton associated to Fuchsian groups is recurrent. Nevertheless, most of our techniques apply in arbitrary hyperbolic groups. We expect that our results should hold (maybe in weaker forms) in this broader context, but the proofs would require significant new ideas. The only results we are aware of in this direction are the following polynomial estimates (that were suggested to us by an anonymous referee): for any symmetric, irreducible, finite-range, aperiodic random walk in an hyperbolic group with spectral radius $R^{-1}$, one has

$$
C R^{-n} n^{-3} \leq p^{n}(1,1) \leq C R^{-n} n^{-1} .
$$

The upper bound follows from the fact that the sequence $R^{2 n} p^{2 n}(1,1)$ is non-increasing and summable, hence $o(1 / n)$. For the lower bound one uses Property RD (see for instance [16], and especially Proposition 1.7 there to get quantitative estimates for hyperbolic groups): there exists a constant $C>0$ such that, for any function $f$ supported in the ball $B(1, n)$, the operator norm of $g \mapsto f \star g$ on $\ell^{2}(\Gamma)$ is bounded by $C n^{3 / 2}\|f\|_{\ell^{2}}$. We apply this estimate to $f(x)=p^{n}(1, x)$ : the norm of the convolution with $f$ is $R^{-n}$, while $\|f\|_{\ell^{2}}=\left(p^{2 n}(1,1)\right)^{1 / 2}$. Therefore, Property RD gives $R^{-n} \leq C n^{3 / 2}\left(p^{2 n}(1,1)\right)^{1 / 2}$, proving the desired lower bound. 
For random walks with non-symmetric step distributions, the local limit theorem holds in free groups. We expect it to hold also in general hyperbolic groups. However, we do not even know how to prove polynomial bounds for this case.

\section{Green's function: preliminaries}

Throughout this section, $X_{n}$ is a symmetric, finite-range irreducible random walk on a finitely generated, nonamenable group $\Gamma$ with (symmetric) generating set $A$. Let $S$ denote the support of the step distribution of the random walk. We assume throughout that $S$ is finite, and hence contained in a ball $B\left(1, C_{0}\right)$ for some $C_{0} \geq 1$.

\subsection{Green's function as a sum over paths}

The Green's function $G_{r}(x, y)$ defined by (1) has an obvious interpretation as a sum over paths from $x$ to $y$. (Note: Here and in the sequel a path in $\Gamma$ is just a sequence $x_{n}$ of vertices in the Cayley graph $G^{\Gamma}$ with $x_{n}^{-1} x_{n+1} \in S$ for all $\left.n\right)$. Denote by $\mathscr{P}(x, y)$ the set of all paths $\gamma$ from $x$ to $y$, and for any such path $\gamma=\left(x_{0}, x_{1}, \ldots, x_{m}\right)$ define the weight

$$
w_{r}(\gamma):=r^{m} \prod_{i=0}^{m-1} p\left(x_{i}, x_{i+1}\right) .
$$

Then

$$
G_{r}(x, y)=\sum_{\gamma \in \mathscr{P}(x, y)} w_{r}(\gamma) .
$$

Since the step distribution $p(x)=p\left(x^{-1}\right)$ is symmetric with respect to inversion, so is the weight function $\gamma \mapsto w_{r}(\gamma)$ : if $\gamma^{R}$ is the reversal of the path $\gamma$, then $w_{r}\left(\gamma^{R}\right)=w_{r}(\gamma)$. Consequently, the Green's function is symmetric in its arguments:

$$
G_{r}(x, y)=G_{r}(y, x) .
$$

Also, the weight function is multiplicative with respect to concatenation of paths, that is, $w_{r}\left(\gamma \gamma^{\prime}\right)=w_{r}(\gamma) w_{r}\left(\gamma^{\prime}\right)$. Since the random walk is irreducible, every generator of the group can be reached by the random walk in finite time with positive probability. It follows that the Green's function satisfies a system of Harnack inequalities: There exists a constant $C<\infty$ such that for each $0<r \leq R$ and all group elements $x, y, z$,

$$
G_{r}(x, z) \leq C^{d(y, z)} G_{r}(x, y) .
$$

Proof of Proposition 1.9. - This is a routine calculation based on the representation (13) of the Green's function as a sum over paths. Since all terms in the power series representation of the Green's function have nonnegative coefficients, interchange of $d / d r$ and $\sum_{\gamma}$ is permissible, so

$$
\frac{d}{d r} G_{r}(x, y)=\sum_{\gamma \in \mathscr{P}(x, y)} \frac{d}{d r} w_{r}(\gamma) .
$$

If $\gamma$ is a path from 1 to $x$ of length $m$, then the derivative with respect to $r$ of the weight $w_{r}(\gamma)$ is $m w_{r}(\gamma) / r$, so $d w_{r}(\gamma) / d r$ contributes one term of size $w_{r}(\gamma) / r$ for each vertex visited by $\gamma$ after its first step. This, together with the multiplicativity of $w_{r}$, yields the identity (9).

4 e SÉRIE - TOME $46-2013$ - No 1 


\subsection{First-passage generating functions}

Other useful generating functions can be obtained by summing path weights over different sets of paths. Two classes of such generating functions that will be used below are the restricted Green's functions and the first-passage generating functions (called the balayage by Ancona [2]) defined as follows. Fix a set of vertices $\Omega \subset \Gamma$, and for any $x, y \in \Gamma$ let $\mathscr{P}(x, y ; \Omega)$ be the set of all paths from $x$ to $y$ that remain in the region $\Omega$ at all except the initial and final points. Define

$$
\begin{aligned}
G_{r}(x, y ; \Omega) & =\sum_{\mathscr{P}(x, y ; \Omega)} w_{r}(\gamma), \quad \text { and } \\
F_{r}(x, y) & =G_{r}(x, y ; \Gamma \backslash\{y\}) .
\end{aligned}
$$

Thus, $F_{r}(x, y)$, the first-passage generating function, is the sum over all paths from $x$ to $y$ that first visit $y$ on the last step. This generating function has the alternative representation

$$
F_{r}(x, y)=E^{x} r^{\tau(y)}
$$

where $\tau(y)$ is the time of the first visit to $y$ by the random walk $X_{n}$, and the expectation extends only over those sample paths such that $\tau(y)<\infty$. Finally, since any visit to $y$ by a path started at $x$ must follow a first visit to $y$,

$$
G_{r}(x, y)=F_{r}(x, y) G_{r}(1,1) .
$$

Therefore, since $G_{r}$ is symmetric in its arguments, so is $F_{r}$.

Lemma 2.1. - We have

$$
\lim _{n \rightarrow \infty} \max _{\{x \in \Gamma:|x|=n\}} G_{R}(1, x)=0 .
$$

Proof. - If $\gamma$ is a path from 1 to $x$, and $\gamma^{\prime}$ a path from $x$ to 1 , then the concatenation $\gamma \gamma^{\prime}$ is a path from 1 back to 1 . Furthermore, since any path from 1 to $x$ or back must make at least $|x| / C_{0}$ steps, the length of $\gamma \gamma^{\prime}$ is at least $2|x| / C_{0}$. Consequently, by symmetry,

$$
F_{R}(1, x)^{2} G_{R}(1,1) \leq \sum_{n=2|x| / C_{0}}^{\infty} P^{1}\left\{X_{n}=1\right\} R^{n} .
$$

Since $G_{R}(1,1)<\infty$, by nonamenability of the group $\Gamma$, the tail-sum on the right side of inequality (17) converges to 0 as $|x| \rightarrow \infty$, and so $F_{R}(1, x) \rightarrow 0$ as $|x| \rightarrow \infty$. Consequently, by (16), so does $G_{R}(1, x)$.

Several variations on this argument will be used later.

\subsection{Subadditivity and the random walk metric}

A path from $x$ to $z$ that visits $y$ can be uniquely split into a path from $x$ to $y$ with first visit to $y$ at the last point, and a path from $y$ to $z$. Consequently, by the Markov property (or alternatively the path representation (13) and the multiplicativity of the weight function $\left.w_{r}\right), G_{r}(x, z) \geq F_{r}(x, y) G_{r}(y, z)$. Since $G_{r}(x, z)=F_{r}(x, z) G_{r}(1,1)$ and $G_{r}(y, z)=F_{r}(y, z) G_{r}(1,1)$, we deduce that the function $-\log F_{r}(x, y)$ is subadditive: 
Lemma 2.2. - For each $r \leq R$ the first-passage generating functions $F_{r}(x, y)$ are supermultiplicative, that is, for any group elements $x, y, z$,

$$
F_{r}(x, z) \geq F_{r}(x, y) F_{r}(y, z) .
$$

Together with Kingman's subadditive ergodic theorem, this implies that the Green's function $G_{r}(1, x)$ must decay at a fixed exponential rate along suitably chosen trajectories. For instance, if

$$
Y_{n}=\xi_{1} \xi_{2} \cdots \xi_{n}
$$

where $\xi_{n}$ is an ergodic Markov chain on the alphabet $A$, or on the set $A^{K}$ of words of length $K$, then Kingman's theorem implies that

$$
\lim n^{-1} \log G_{r}\left(1, Y_{n}\right)=\alpha \quad \text { a.s. }
$$

where $\alpha$ is a constant depending only on $r$ and the transition probabilities of the underlying Markov chain. More generally, if $\xi_{n}$ is a suitable ergodic stationary process, then (18) will hold. Super-multiplicativity of the Green's function also implies the following.

COROLlary 2.3. - The function $d_{G}(x, y):=\log F_{R}(x, y)$ is a metric on $\Gamma$.

Proof. - The triangle inequality is immediate from Lemma 2.2, and symmetry $d_{G}(x, y)=d_{G}(y, x)$ follows from the corresponding symmetry property (14) of the Green's function. Thus, to show that $d_{G}$ is a metric (and not merely a pseudo-metric) it suffices to show that if $x \neq y$ then $F_{R}(x, y)<1$. But this follows from the fact (2) that the Green's function is finite at the spectral radius, because the path representation implies that

$$
G_{R}(x, x) \geq 1+F_{R}(x, y)^{2}+F_{R}(x, y)^{4}+\cdots .
$$

Call $d_{G}$ the Green metric associated with $F_{R}$. The Green metric associated with $F_{1}$ has been used by a number of authors, for instance in [7], [8] and [9]. The Harnack inequalities imply that $d_{G}$ is dominated by a constant multiple of the word metric $d$. In general, there is no domination in the other direction, unless one puts additional restrictions on the Green function:

Proposition 2.4. - If the Green's function decays exponentially in $d(x, y)$ (that is, if inequality (6) holds for all $x, y \in \Gamma$ ), then the Green metric $d_{G}$ and the word metric $d$ on $\Gamma$ are equivalent, that is, there are constants $0<C_{1}<C_{2}<\infty$ such that for all $x, y \in \Gamma$,

$$
C_{1} d(x, y) \leq d_{G}(x, y) \leq C_{2} d(x, y) \text {. }
$$

Even when the group $\Gamma$ is hyperbolic, this proposition does not imply in general that $d_{G}$ is hyperbolic, since the quasi-isometry invariance of hyperbolicity only holds for geodesic spaces, while $\left(\Gamma, d_{G}\right)$ is not geodesic in general. Nevertheless, Theorem 1.1 in [9] shows that $d_{G}$ is hyperbolic when Ancona's inequalities (5) are satisfied for $r=R$.

4 e SÉRIE - TOME $46-2013$ - No 1 


\subsection{Green's function and branching random walks}

There is a simple interpretation of the Green's function $G_{r}(x, y)$ in terms of the occupation statistics of branching random walks. A branching random walk is built using a probability distribution $Q=\left\{q_{k}\right\}_{k \geq 0}$ on the nonnegative integers, called the offspring distribution, together with the step distribution $\mathscr{P}:=\left\{p(x, y)=p\left(x^{-1} y\right)\right\}_{x, y \in \Gamma}$ of the underlying random walk, according to the following rules: At each time $n \geq 0$, each particle fissions and then dies, creating a random number of offspring with distribution $Q$; the offspring counts for different particles are mutually independent. Each offspring particle then moves from the location of its parent by making a random jump according to the step distribution $p(x, y)$; the jumps are once again mutually independent. Consider the initial condition which places a single particle at site $x \in \Gamma$, and denote the corresponding probability measure on population evolutions by $Q^{x}$.

Proposition 2.5. - Under $Q^{x}$, the total number of particles in generation $n$ evolves as a Galton-Watson process with offspring distribution Q. If the offspring distribution has mean $r \leq R$, then under $Q^{x}$ the expected number of particles at location y at time $n$ is $r^{n} P^{x}\left\{X_{n}=y\right\}$, where under $P^{x}$ the process $X_{n}$ is an ordinary random walk with step distribution $\mathscr{P}$. Therefore, $G_{r}(x, y)$ is the mean total number of particle visits to location $y$.

Proof. - The first assertion follows easily from the definition of a Galton-Watson process - see [4] for the definition and basic theory. The second is easily proved by induction on $n$. The third then follows from the Formula (1) for the Green's function.

There are similar interpretations of the restricted Green's function $G_{r}(x, y ; \Omega)$ and the first-passage generating function $F_{r}(x, y)$. In particular, if particles of the branching random walk are allowed to reproduce only in the region $\Omega$, then $G_{r}(x, y ; \Omega)$ is the mean number of particle visits to $y$ in this modified branching random walk.

\section{Exponential decay of the Green's function}

\subsection{Hyperbolic geometry}

In this section, we prove that the Green's function of a symmetric random walk on a cocompact Fuchsian group decays exponentially. The proof is most conveniently formulated using some basic ingredients of hyperbolic geometry (see, e.g., [24], chs. 3-4).

For any co-compact Fuchsian group $\Gamma$ there is a fundamental polygon $\mathcal{F}$ for the action of $\Gamma$. The closure of $\mathcal{F}$ is a compact, finite-sided polygon whose sides are arcs of hyperbolic geodesics. The hyperbolic disk $\mathbb{D}$ is tiled by the images $x \mathcal{F}$ of $\mathcal{F}$, where $x \in \Gamma$; distinct elements $x, y \in \Gamma$ correspond to tiles $x \mathcal{F}$ and $y \mathcal{F}$ which intersect, if at all, in a single side or vertex of each tile. Thus, the elements of $\Gamma$ are in bijective correspondence with the tiles of the tessellation, or alternatively with the points $x O$ in the $\Gamma$-orbit of a distinguished point $O \in \mathcal{F}^{\circ}$. The Cayley graph of $\Gamma$ (relative to the standard generators) is gotten by putting edges between those vertices $x, y \in \Gamma$ such that the tiles $x \mathcal{F}$ and $y \mathcal{F}$ share a side. The word metric $d=d_{\Gamma}$ on $\Gamma$ is defined to be the (Cayley) graph distance. The hyperbolic metric induces another distance $d_{\mathbb{H}}(x, y)$ on $\Gamma$, defined to be the hyperbolic distance between the points $x O$ and $y O$. The metrics $d$ and $d_{\mathbb{H}}$ are both left-invariant. 
The following fact is well known (see, e.g., [12], Theorem 1).

Lemma 3.1. - The restriction of the hyperbolic metric $d_{\mathbb{H}}$ to $\Gamma$ and the word metric $d$ on $\Gamma$ are equivalent.

\subsection{Barriers}

To prove that the Green's function decays exponentially fast, we will construct barriers along the hyperbolic geodesic between two points $x O$ and $y O$ in such a way that the total weight $w_{R}$ attached to paths crossing any of those barriers is small. Recall that a path in $\Gamma$ is a sequence $x_{i}$ such that successive jumps $x_{n}^{-1} x_{n+1}$ are all elements of $S$, the support of the step distribution of the random walk. Recall also that $S$ is symmetric and contained in the ball $B\left(1, C_{0}\right)$ of radius $C_{0}$ centered at the group identity 1 . A halfplane is a subset of $\mathbb{D}$ (or its intersection with $\Gamma O$ ) whose boundary is a doubly infinite hyperbolic geodesic.

Definition 3.2. - A barrier is a triple $(V, W, B)$ consisting of nonoverlapping halfplanes $V, W \subset \mathbb{D}$ and a subset $B \subset \Gamma$ disjoint from $V \cup W$ such that every path in $\Gamma O$ from $V$ to $W$ must pass through $B$, and

$$
\max _{x \in V} \sum_{b \in B} G_{R}(x, b) \leq \frac{1}{2} .
$$

For distinct points $\xi, \zeta \in \partial \mathbb{D}$, a barrier between $\xi$ and $\zeta$ is a barrier $(V, W, B)$ such that $\xi$ and $\zeta$ are interior points of the $\operatorname{arcs} \operatorname{cl}(V) \cap \partial \mathbb{D}$ and $\operatorname{cl}(W) \cap \partial \mathbb{D}$, respectively. (We also say that such a barrier $(V, W, B)$ separates $\xi$ and $\zeta$.)

Observe that if $(V, W, B)$ is a barrier then the distance between the hyperbolic geodesics bounding $V$ and $W$ is positive: in particular, these geodesics cannot have a common endpoint on $\partial \mathbb{D}$. Moreover, the set $B$ must have limit points in each of the two arcs of $\partial \mathbb{D}$ separating $\operatorname{cl}(V) \cap \partial \mathbb{D}$ and $\operatorname{cl}(W) \cap \partial \mathbb{D}$. Also, if $(V, W, B)$ is a barrier then (i) for any $g \in \Gamma$ the translate $(g V, g W, g B)$ is a barrier, and (ii) if $V^{\prime}, W^{\prime}$ are halfplanes contained in $V, W$, respectively, then $\left(V^{\prime}, W^{\prime}, B\right)$ is a barrier.

Theorem 3.3. - For any two points $\xi \neq \eta \in \partial \mathbb{D}$, there exists a barrier between $\xi$ and $\eta$.

Before turning to the proof, we record an easy consequence.

COROLlary 3.4. - There exists $K<\infty$ such that for any two hyperbolic geodesics $\alpha, \beta$ at distance $K$ or greater there is a barrier $(A, B, C)$ such that the halfplanes $A$ and $B$ are bounded by $\alpha$ and $\beta$, respectively.

Proof. - Let $Q$ be the set of all pairs of distinct points $(\xi, \zeta) \in \partial \mathbb{D}$ such that the geodesic with endpoints $\xi$ and $\zeta$ enters the interior of the fundamental polygon $\mathcal{F}$. By Theorem 3.3, any such pair $\xi, \zeta$ is separated by a barrier $(V, W, B)$; the halfplanes $V, W$ can be chosen to have arbitrarily small (Euclidean) diameters, and so in particular we can assume that neither $V$ nor $W$ intersects $\mathcal{F}$. If $(\xi, \zeta) \in Q$ are separated by the barrier $(V, W, B)$ then all nearby pairs $\left(\xi^{\prime}, \zeta^{\prime}\right)$ are also separated by this barrier. Since $Q$ has compact closure in $\partial \mathbb{D} \times \partial \mathbb{D}$, it follows that there is a finite set of barriers $\left(V_{i}, W_{i}, B_{i}\right)$ and a constant $\delta>0$ such that (i) for any pair $(\xi, \zeta) \in Q$ at least one of the barriers separates $\xi$ from $\zeta$, and (ii) for this barrier $\left(V_{i}, W_{i}, B_{i}\right)$ the Euclidean circles of radius $\delta$ centered at $\xi$ and $\zeta$ intersect $\mathbb{D}$ only inside the 
halfplanes $V_{i}$ and $W_{i}$, respectively. Furthermore, the barriers can be chosen so that none of the halfplanes $V_{i}, W_{i}$ intersects the fundamental polygon $\mathcal{F}$.

Suppose that $\alpha, \beta$ are hyperbolic geodesics at distance $D$ greater than four times the diameter of $\mathcal{F}$. Let $\gamma$ be the geodesic segment of minimal hyperbolic length with endpoints on $\alpha$ and $\beta$, and let $M$ be the midpoint of this segment. Then there is an element $g \in \Gamma$ that maps the geodesic segment $\gamma$ to a segment $g \gamma$ which crosses $\mathcal{F}$ in such a way that the midpoint $g M$ lies in one of the tiles $h \mathcal{F}$ within distance $\operatorname{diam}(\mathcal{F})$ of $\mathcal{F}$. Let $\xi, \zeta$ be the endpoints on $\partial \mathbb{D}$ of the doubly infinite geodesic extension of $g \gamma$. By construction, the pair $(\xi, \zeta)$ is an element of $Q$, so $\xi$ and $\zeta$ are separated by one of the barriers $\left(V_{i}, W_{i}, B_{i}\right)$. If the distance $D$ between $\alpha$ and $\beta$ is sufficiently large, say $D \geq K$, then the geodesics $g \alpha$ and $g \beta$ will be entirely contained in the halfplanes $V_{i}$ and $W_{i}$, respectively. (This is because the midpoint $g M$ of the connecting segment $g \gamma$ lies within distance $\operatorname{diam}(\mathcal{F})$ of $\mathcal{F}$, so both $g \alpha$ and $g \beta$ are at distance greater than $D / 2-2 \operatorname{diam}(\mathcal{F})$ from $\mathcal{F}$, and consequently, if $D$ is sufficiently large, must lie inside circles of Euclidean radius $\delta$ centered at points on $\partial \mathbb{D}$.) If $g \alpha$ and $g \beta$ are contained in $V_{i}$ and $W_{i}$, then there is a barrier $(g A, g B, g C)$ such that the halfplanes $g A$ and $g B$ are bounded by $g \alpha$ and $g \beta$. Finally, since translates of barriers are barriers, it follows that $(A, B, C)$ is a barrier with the desired property.

The rest of this subsection is devoted to the proof of Theorem 3.3. The strategy is to construct the barrier using typical trajectories of the random walk.

Lemma 3.5. - There exist $C>0$ and $\varrho<1$ such that $P^{1}\left(G_{R}\left(1, X_{n}\right) \geq \varrho^{n}\right) \leq C \varrho^{n}$.

Proof. - Since $G_{r}(1, x)=F_{r}(1, x) G_{r}(1,1)$, it suffices to prove the corresponding statement where the Green's function $G_{R}\left(1, X_{n}\right)$ is replaced by the first-passage generating function $F_{R}\left(1, X_{n}\right)$. Now any path $\gamma$ of length $n$ from 1 to a point $x$ can be concatenated with any path $\gamma^{\prime}$ from $x$ to 1 , yielding a path from 1 to itself. If the path $\gamma^{\prime}$ does not re-visit the point $x$ the splitting $\left(\gamma, \gamma^{\prime}\right)$ of the concatenated path is a last-exit decomposition, so the mapping $\left(\gamma, \gamma^{\prime}\right) \mapsto \gamma \gamma^{\prime}$ is injective. Hence, summing the weights of all such paths gives a lower bound for $G_{R}(1,1)$ : using the symmetry $F_{R}(x, 1)=F_{R}(1, x)$, we obtain

$$
G_{R}(1,1) \geq \sum_{x} R^{n} P^{1}\left(X_{n}=x\right) F_{R}(1, x)=R^{n} E^{1}\left(F_{R}\left(X_{n}, 1\right)\right) .
$$

Therefore,

$$
P^{1}\left(F_{R}\left(X_{n}, 1\right) \geq \varrho^{n}\right) \leq \frac{1}{\varrho^{n}} E^{1}\left(F_{R}\left(X_{n}, 1\right)\right) \leq C R^{-n} \varrho^{-n},
$$

and so the desired inequality holds with $\varrho=R^{-1 / 2}$.

Lemma 3.6. - For almost all independent trajectories $X_{0}=1, X_{1}, \ldots$ and $Y_{0}=1, Y_{1}, \ldots$ of the random walk,

$$
\sum_{m, n \in \mathbb{N}} G_{R}\left(X_{m}, Y_{n}\right)<\infty
$$


Proof. - For fixed $m$ and $n, Y_{n}^{-1} X_{m}$ is distributed as $X_{n+m}$, by symmetry of the step distribution. Therefore,

$$
\begin{aligned}
P\left(G_{R}\left(X_{m}, Y_{n}\right) \geq \varrho^{m+n}\right)=P\left(G_{R}\left(Y_{n}^{-1} X_{m}, 1\right)\right. & \left.\geq \varrho^{m+n}\right) \\
& =P\left(G_{R}\left(X_{n+m}, 1\right) \geq \varrho^{m+n}\right) \leq C \varrho^{n+m},
\end{aligned}
$$

by the previous lemma. Since this quantity is summable in $m$ and $n$, Borel-Cantelli ensures that, almost surely, $G_{R}\left(X_{m}, Y_{n}\right) \leq \varrho^{m+n}$ for all but finitely many pairs $(m, n)$.

We will construct barriers by first constructing pre-barriers as defined below.

Definition 3.7. - A pre-barrier is a quadruple $(V, W, A, B)$ of pairwise disjoint sets such that $V, W$ are non-overlapping halfplanes and $A, B$ are subsets of $\Gamma$ such that

(i) every path in $\Gamma$ from $V$ to $W$ must enter $A \cup B$, with first entrance at a point $a \in A$ and last exit at a point $b \in B$

(ii) every path in $\Gamma$ from $V$ to $B$ must pass through $A$; and

(iii) we have

$$
\sum_{a \in A, b \in B} G_{R}(a, b)<\infty
$$

A pre-barrier $(V, W, A, B)$ separates points $\xi, \zeta \in \partial \mathbb{D}$ if $\xi$ and $\zeta$ are interior points of the arcs $\operatorname{cl}(V) \cap \partial \mathbb{D}$ and $\operatorname{cl}(W) \cap \partial \mathbb{D}$, respectively.

Lemma 3.8. - For any two distinct points $\xi, \eta \in \partial \mathbb{D}$, there exists a pre-barrier separating $\xi$ and $\eta$.

Proof. - Choose two disjoint compact subintervals $J$ and $K$ in one of the connected components of $\partial \mathbb{D}-\{\xi, \eta\}$ such that $J$ is closer to $\xi$ than $K$, and similarly choose $J^{\prime}, K^{\prime}$ in the other connected component of $\partial \mathbb{D}-\{\xi, \eta\}$.

Let $X_{n}, X_{n}^{\prime}, Y_{n}, Y_{n}^{\prime}$ be independent copies of the random walk starting from 1 , and let $\Omega^{\prime}$ be the event that $X_{n}$ converges to $J, X_{n}^{\prime}$ converges to $J^{\prime}, Y_{n}$ converges to $K$ and $Y_{n}^{\prime}$ converges to $K^{\prime}$. The Poisson boundary of the random walk is identified with the topological boundary $S^{1}$, and the limiting measure has full support there since the group action is minimal on the boundary. Therefore, $\Omega^{\prime}$ has positive probability. Moreover, Lemma 3.6 implies that almost surely $\sum G_{R}\left(\tilde{X}_{m}, \tilde{Y}_{n}\right)<\infty$ for $\tilde{X}=X$ or $X^{\prime}$ and $\tilde{Y}=Y$ or $Y^{\prime}$. Consequently, there exist two-sided infinite paths $\left\{x_{n}\right\}_{n \in \mathbb{Z}}$ connecting $J$ with $J^{\prime}$ and $\left\{y_{n}\right\}_{n \in \mathbb{Z}}$ connecting $K$ with $K^{\prime}$ such that $\sum_{m, n \in \mathbb{Z}} G_{R}\left(x_{m}, y_{n}\right)<\infty$. Since $J \cup J^{\prime}$ is disjoint from $K \cup K^{\prime}$, the trajectories $x_{m}$ and $y_{n}$ are disjoint for large $|m|,|n|$. Modifying these trajectories at finitely many places (which does not change the validity of $\sum_{m, n} G_{R}\left(x_{m}, y_{n}\right)<\infty$ ) and then replacing each point of the trajectory by a ball of radius $2 C_{0}$ (so that a trajectory of the random walk can not jump past the newly constructed set), we obtain the required pre-barrier.

Theorem 3.3 follows from the previous lemma and the next lemma.

Lemma 3.9. - If $(V, W, A, B)$ is a pre-barrier separating $\xi$ and $\eta$, then there exist halfplanes $V^{\prime} \subseteq V$ and $W^{\prime} \subseteq W$ such that $\left(V^{\prime}, W^{\prime}, B\right)$ is a barrier separating $\xi$ and $\eta$.

$4^{\mathrm{e}}$ SÉRIE - TOME $46-2013-\mathrm{N}^{\circ} 1$ 
Proof. - The only nontrivial point is the existence of a halfplane neighborhood $V^{\prime}$ of $\xi$ such that $\max _{x \in V^{\prime}} \sum_{b \in B} G_{R}(x, b) \leq 1 / 2$.

Let $V^{\prime}$ be a small neighborhood of $\xi$. Every path from $V^{\prime}$ to $B$ must go first through $A$. Decomposing such a path according to the first visited point in $A$, we find that for any $b \in B$,

$$
\begin{aligned}
G_{R}(x, b) & \leq \sum_{a \in A} G_{R}(x, a) G_{R}(a, b) \\
& \leq \sup _{a \in A} G_{R}(x, a) \cdot \sum_{a \in A, b \in B} G_{R}(a, b) \\
& \leq C \sup _{a \in A} G_{R}(x, a) .
\end{aligned}
$$

By Lemma 2.1, $G_{R}(1, x)$ tends uniformly to 0 when $|x| \rightarrow \infty$. Therefore, if $V^{\prime}$ is small enough then $\sup _{a \in A} G_{R}(x, a)$ is smaller than $1 /(2 C)$.

\subsection{Exponential decay of the Green's function}

THEOREM 3.10. - The Green's function $G_{R}(x, y)$ of a symmetric, irreducible, finite-range random walk on a co-compact Fuchsian group $\Gamma$, evaluated at its radius of convergence $R$, decays exponentially in the distance $d(x, y)$. In particular, there exist constants $C<\infty$ and $\varrho<1$ such that for every $x \in \Gamma$,

$$
G_{R}(1, x) \leq C \varrho^{|x|}
$$

To prove this theorem, we will show that disjoint barriers can be placed consecutively along the hyperbolic geodesic from 1 to $x$ (identified with $O$ and $x O$ ) in $\mathbb{D}$.

Lemma 3.11. - There exists $L>0$ with the following property. If $x \in \Gamma$ satisfies $d(1, x) \geq n L$ for some integer $n \geq 2$, then there exist $n$ barriers $\left(V_{i}, W_{i}, B_{i}\right)$ such that

(i) the sets $B_{i}$ are pairwise disjoint;

(ii) every path from 1 to $x$ goes successively through $B_{0}, B_{1}, B_{2}, \ldots, B_{n-1}$;

(iii) we have $\sum_{b_{0} \in B_{0}} G_{R}\left(1, b_{0}\right) \leq 1 / 2$; and

(iv) for any $i<n-1$ and any $b_{i} \in B_{i}$,

$$
\sum_{b_{i+1} \in B_{i+1}} G_{R}\left(b_{i}, b_{i+1}\right) \leq 1 / 2 .
$$

Proof. - This is an easy consequence of Corollary 3.4 and Lemma 3.1. Let $\gamma$ be the hyperbolic geodesic segment from 1 to $x$; by Lemma 3.1, if $L$ is sufficiently large then the hyperbolic length of $\gamma$ is at least $n K$. Let $\beta_{0}, \beta_{1}, \ldots \beta_{n}$ be the hyperbolic geodesics that cross $\gamma$ orthogonally at the points $1, y_{1}, y_{2}, \ldots y_{n}$ at distances $0, K, 2 K, \ldots, n K$ from the endpoint 1. Corollary 3.4 implies that for each $0 \leq i<n$ there is a barrier $\left(V_{i}, W_{i}, B_{i}\right)$ such that $V_{i}$ is bounded by $\beta_{i}$ and $W_{i}$ by $\beta_{i+1}$. These have all the desired properties. 
Proof of Theorem 3.10. - Let $x \in \Gamma$ be a vertex such that $d(1, x) \in[n L,(n+1) L)$. Decomposing paths from 1 to $x$ according to their first points of entry into $B_{0}$, then $B_{1}$, and so on, we get

$$
\begin{aligned}
G_{R}(1, x) & \leq \sum_{b_{0} \in B_{0}, \ldots, b_{n-1} \in B_{n-1}} G_{R}\left(1, b_{0}\right) G_{R}\left(b_{0}, b_{1}\right) \ldots G_{R}\left(b_{n-2}, b_{n-1}\right) G_{R}\left(b_{n-1}, x\right) \\
& \leq 2^{-n+1} \sup _{b_{n-1} \in B_{n-1}} G_{R}\left(b_{n-1}, x\right) .
\end{aligned}
$$

Since $G_{R}$ tends to 0 at infinity, it is uniformly bounded, and so the exponential bound follows.

\section{Ancona's inequalities}

In this section, we shall prove Ancona's inequalities (5) and extend them to relative Green's functions (Theorem 4.1). We will then show how these inequalities are used to identify the Martin boundary and to establish the Hölder continuity of the Martin kernel. The proofs of the various Ancona inequalities will rely on the exponential decay of the Green's function, which was proved in Section 3, and the hyperbolic character of the Cayley graph. Recall that a finitely generated group $\Gamma$ (or alternatively its Cayley graph) is hyperbolic in the sense of Gromov [20] if it satisfies the thin triangle property, that is, there exists a constant $\delta<\infty$ such that for any geodesic triangle $T$ in $\Gamma$, each point on a side of $T$ is within distance $\delta$ of at least one of the other two sides. We then say that $\delta$ is a Gromov constant for $\Gamma$. The minimal Gromov constant $\delta$ will in general depend on the choice of the generating set; however, if $\Gamma$ is hyperbolic with respect to one (finite) set of generators then it is hyperbolic with respect to every finite set of generators.

\subsection{Relative Ancona's inequalities}

For technical reasons, we will need an extension of Ancona's inequalities valid for relative Green's functions (as defined in Subsection 2.2). These inequalities assert that, for any point $z$ on a geodesic segment between two points $x$ and $y$, and for a suitable class of domains $\Omega$, one has

$$
G_{r}(x, y ; \Omega) \leq C G_{r}(x, z ; \Omega) G_{r}(z, y ; \Omega) .
$$

The constant $C$ should be independent of $x, y, z$, of $\Omega$, and of $r \in[1, R]$. The usual Ancona inequalities (5) correspond to the case $\Omega=\Gamma$. Clearly, inequality (21) cannot hold for all domains $\Omega$, because in general there might not be positive-probability paths from $x$ to $z$ in $\Omega$. Therefore, some restrictions on the set $\Omega$ are necessary.

Relative versions of Ancona's inequalities play an important role in [1] (see th. 1' there), $[30,22]$. In the latter two papers, such inequalities are proved for any domain $\Omega$ that contains a neighborhood of fixed size $C$ of the geodesic segment $[x y]$. We are only able to deal with a smaller class of domains, but these will nevertheless be sufficient for our purposes.

THEOREM 4.1. - For any symmetric, irreducible, finite-range random walk on a co-compact Fuchsian group $\Gamma$ there exist positive constants $C$ and $C^{\prime}$ such that the following statement holds.

$4^{\mathrm{e}}$ SÉRIE - TOME $46-2013-\mathrm{N}^{\circ} 1$ 
For any geodesic segment $[x y]$ and any $z \in[x y]$, and for any subset $\Omega \subset \Gamma$ with the property that for each $w \in[x y]$ the ball of radius $C^{\prime}+d(w, z) / 2$ centered at $w$ is contained in $\Omega$,

$$
G_{r}(x, y ; \Omega) \leq C G_{r}(x, z ; \Omega) G_{r}(z, y ; \Omega) \quad \text { for all } 1 \leq r \leq R .
$$

There is another natural class of domains $\Omega$ for which we can establish relative Ancona's inequalities (under additional assumptions on the random walk).

Definition 4.2. - A set $\Omega \subset \Gamma$ is convex if for all pairs $x, y \in \Omega$ there is a geodesic segment from $x$ to $y$ contained in $\Omega$.

Theorem 4.3. - Consider an irreducible, symmetric random walk on a co-compact Fuchsian group $\Gamma$, and assume that its step distribution gives positive probability to any element of the generating set used to define the word distance. There exists a constant $C>0$ such that, for every convex set $\Omega$ containing the geodesic segment $[x y]$, and for every point $z \in[x y]$, the Ancona inequality (22) holds.

The converse inequality $G_{r}(x, y ; \Omega) \geq C G_{r}(x, z ; \Omega) G_{r}(z, y ; \Omega)$ is trivial (see Lemma 2.2), so the theorems really say that $G_{r}(x, y ; \Omega) \asymp G_{r}(x, z ; \Omega) G_{r}(z, y ; \Omega)$ (meaning that the ratio between the two sides of this equation remains bounded away from 0 and $\infty$ ).

The proof of the two theorems will use the following lemma, which is an easy consequence of the exponential decay of the Green's function and the geometry of the hyperbolic disk.

Lemma 4.4. - There exist $C>0$ and $\alpha>0$ such that, for any geodesic segment [xy], any $z \in[x y]$ and any $k \geq 0$,

$$
G_{R}\left(x, y ; B_{k}(z)^{c}\right) \leq C \exp \left\{-e^{\alpha k}\right\} .
$$

Here $B_{k}(z)^{c}$ is the complement of the ball $B_{k}(z)$ of radius $k$ centered at $z$. Since the distance from $x$ to $y$ in the complement of $B_{k}(z)$ grows exponentially with $k$, and the Green's function itself decays exponentially, the double exponential bound in the conclusion of the lemma should not be surprising.

Proof. - It suffices to prove the lemma for large $k$. Since the Green's function is translation invariant, we can assume without loss of generality that $z=1$. (Recall that $1 \in \Gamma$ is identified with the point $O \in \mathbb{D}$.) Consider the hyperbolic circle $\mathscr{C}$ of radius $k / C$ around $O$, where $C$ is sufficiently large that this circle is contained in the ball $B_{k}(1)$ for the Cayley graph distance. Since the hyperbolic length of $\mathscr{C}$ grows exponentially with $k$, one can set along this circle an exponential number $e^{\alpha k}$ of geodesic rays $D_{i}$ starting from $O$ and going to infinity, such that the minimum distance $d$ between any successive rays $D_{i}$ and $D_{i+1}$ along $\mathscr{C}$ is arbitrarily large (the exponential rate $\alpha>0$ will not depend on $d$, but the minimum radius $k / C$ will.). Let $\tilde{D}_{i}$ be a thickening of $D_{i}$ so that a path making jumps of length at most $C_{0}$ can not jump across $\tilde{D}_{i}$. If $d$ is large enough then $\sum_{a_{i} \in \tilde{D}_{i}, a_{i+1} \in \tilde{D}_{i+1}} G_{R}\left(a_{i}, a_{i+1}\right) \leq 1 / 2$ (this sum is dominated by a geometric series, thanks to the exponential decay of the Green's function, and it has arbitrarily small first term if $d$ is large). A path from $x$ to $y$ that avoids $B_{k}(1)$ has to go around the circle in one direction or the other, and will therefore cross at least half the domains $\tilde{D}_{i}$. As in the conclusion of the proof of Theorem 3.10, this yields $G_{R}\left(x, y ; B_{k}(1)^{c}\right) \leq C 2^{-e^{\alpha k} / 2}$. 
Proof of Theorems 4.1-4.3. - Let $[x y]$ be a geodesic segment of some length $m$ (relative to the Cayley graph distance) with endpoints $x, y \in \Gamma$, let $z \in \Gamma$ be a point on [xy], and let $\Omega$ be a domain containing $[x y]$ satisfying the assumptions of Theorem 4.1 or 4.3.

We first construct by induction points $x_{n}, y_{n}$ on the geodesic segment $[x y]$ in such a way that for each $n$ the points $x_{n}, z, y_{n}$ occur in order on $[x y]$. Start by setting $x_{0}=x$ and $y_{0}=y$. At step $n$, if $z$ is in the left half of $\left[x_{n} y_{n}\right]$ then set $x_{n+1}=x_{n}$ and let $y_{n+1}$ be a point in $\left[x_{n} y_{n}\right]$ such that $\left|d\left(y_{n+1}, y_{n}\right)-d\left(x_{n}, y_{n}\right) / 4\right| \leq 1$. Similarly, if $z$ is in the right half of $\left[x_{n} y_{n}\right]$ then define $y_{n+1}=y_{n}$ and let $x_{n+1}$ be a point on $\left[x_{n} y_{n}\right]$ such that $\left|d\left(x_{n}, x_{n+1}\right)-d\left(x_{n}, y_{n}\right) / 4\right| \leq 1$. The construction ends at the first step $N$ such that $d\left(x_{N}, y_{N}\right) \leq A$, for some large $A>0$. By construction, for all $n \leq N$,

$$
d\left(x_{n}, y_{n}\right)=(3 / 4)^{n} m+O(1) \text { and } z \in\left[x_{n} y_{n}\right],
$$

where the $O(1)$ term is bounded by $\sum_{k=0}^{\infty}(3 / 4)^{k}=4$.

At each step of the construction, the discarded interval ( $\left[y_{n+1} y_{n}\right]$ in the first case, $\left[x_{n} x_{n+1}\right]$ in the second) lies either to the right or to the left of $z$ on the interval $[x y]$. In either case, define $B_{n+1}$ to be the ball of radius $d\left(x_{n}, y_{n}\right) / 100$ centered at the midpoint of the discarded interval. Observe that these balls are pairwise disjoint. For each $n$, let $a_{n}$ and $b_{n}$ be the midpoints of the last discarded intervals to the left and right of $z$ respectively (with the convention that if no intervals to the left of $z$ have been discarded by step $n$ then $a_{n}=x$, and similarly if no intervals to the right have been discarded then $b_{n}=y$ ). By construction,

$$
a_{n} \leq x_{n} \leq z \leq y_{n} \leq b_{n}
$$

in the natural ordering (left to right) on $[x y]$, and so the center of the ball $B_{n+1}$ must lie on the segment $\left[a_{n} b_{n}\right]$. Furthermore, by hyperbolicity, if $u_{n}, v_{n}$ are any points in the balls $B_{l}, B_{r}$ closest to $z$ on the left and right, respectively, centered at points in intervals removed by step $n$, then any geodesic segment $\left[u_{n} v_{n}\right]$ connecting $u_{n}$ and $v_{n}$ must pass within distance $2 \delta$ of $z$, where $\delta$ is a Gromov constant for the Cayley graph (provided the constant $A$ that determines the termination point of the construction is sufficiently large). In fact, the entire geodesic segment $\left[x_{n} y_{n}\right]$ must lie within distance $2 \delta$ of $\left[u_{n} v_{n}\right]$.

We now decompose $G_{r}(x, y ; \Omega)$ by splitting random walk trajectories at visits to the balls $B_{i}$. Begin with $u_{0}=x$ and $v_{0}=y$. If $z$ is in the first half of $\left[u_{0} v_{0}\right]$, split paths at the last visit to $B_{1}$; this yields

$$
G_{r}\left(u_{0}, v_{0} ; \Omega\right)=G_{r}\left(u_{0}, v_{0} ; \Omega \cap B_{1}^{c}\right)+\sum_{v_{1} \in B_{1} \cap \Omega} G_{r}\left(u_{0}, v_{1} ; \Omega\right) G_{r}\left(v_{1}, v_{0} ; \Omega \cap B_{1}^{c}\right) .
$$

If $z$ is in the second half of $\left[u_{0} v_{0}\right]$, split paths at the first visit to $B_{1}$; this gives

$$
G_{r}\left(u_{0}, v_{0} ; \Omega\right)=G_{r}\left(u_{0}, v_{0} ; \Omega \cap B_{1}^{c}\right)+\sum_{u_{1} \in B_{1} \cap \Omega} G_{r}\left(u_{0}, u_{1} ; \Omega \cap B_{1}^{c}\right) G_{r}\left(u_{1}, v_{0} ; \Omega\right) .
$$

To get manageable formulas, we will introduce a more symmetric notation. Let $H(u, v ; B)=G_{r}(u, v ; \Omega \cap B)$ if $u \neq v$, and 1 if $u=v$. In addition, write $u_{1}=u_{0}$ in the first case (that is, if $z$ is in the first half of $\left[u_{0} v_{0}\right]$ ), and write $v_{1}=v_{0}$ in the second case. The formulas above become

$$
G_{r}\left(u_{0}, v_{0} ; \Omega\right)=H\left(u_{0}, v_{0} ; B_{1}^{c}\right)+\sum_{u_{1}, v_{1}} H\left(u_{0}, u_{1} ; B_{1}^{c}\right) G_{r}\left(u_{1}, v_{1} ; \Omega\right) H\left(v_{1}, v_{0} ; B_{1}^{c}\right) .
$$


This procedure can be iterated. The factor $G_{r}\left(u_{1}, v_{1} ; \Omega\right)$ can be decomposed by splitting random walk trajectories at visits to $B_{2}$; this leads to a second sum with an inner factor $G_{r}\left(u_{2}, v_{2} ; \Omega\right)$. This factor can again be decomposed, and so on, whence we obtain, by induction, for any $k \leq N$,

$$
\begin{aligned}
G_{r}\left(u_{0}, v_{0} ; \Omega\right)= & \sum_{j=0}^{k-1} \sum_{\substack{u_{1}, \ldots, u_{j} \\
v_{1}, \ldots, v_{j}}} H\left(u_{j}, v_{j} ; B_{j+1}^{c}\right) \prod_{i=0}^{j-1}\left\{H\left(u_{i}, u_{i+1} ; B_{i+1}^{c}\right) H\left(v_{i+1}, v_{i} ; B_{i+1}^{c}\right)\right\} \\
& +\sum_{\substack{u_{1}, \ldots, u_{k} \\
v_{1}, \ldots, v_{k}}} G_{r}\left(u_{k}, v_{k} ; \Omega\right) \prod_{i=0}^{k-1}\left\{H\left(u_{i}, u_{i+1} ; B_{i+1}^{c}\right) H\left(v_{i+1}, v_{i} ; B_{i+1}^{c}\right)\right\} .
\end{aligned}
$$

For each index $i$ in the products, either $u_{i}=u_{i+1}$ or $v_{i}=v_{i+1}$, and the corresponding $H$-factor is 1 . In the first case, $v_{i+1} \in B_{i+1} \cap \Omega$; in the second case, $u_{i+1} \in B_{i+1} \cap \Omega$. Thus, for each $i$ the points $u_{i}, v_{i}$ must lie in the balls $B_{l}, B_{r}$ centered at the midpoints of the nearest discarded (by step $i$ ) intervals to the left and right of $z$. As noted earlier, this implies that any geodesic segment $\left[u_{i} v_{i}\right]$ must pass within distance $2 \delta$ of $z$. Moreover, since the center of the ball $B_{i+1}$ lies on $\left[x_{i} y_{i}\right]$, the ball contains a ball with center on $\left[u_{i} v_{i}\right]$ of radius $\left(m(3 / 4)^{i}-4\right) / 100-2 \delta$. Therefore, by Lemma 4.4, for suitable constants $C, \beta>0$,

$$
H\left(u_{i}, v_{i} ; B_{i+1}^{c}\right) \leq C \exp \left\{-e^{\beta m(3 / 4)^{i}}\right\} .
$$

On the other hand, under the hypotheses of Theorem 4.1 (for sufficiently large $C^{\prime}$ ), a fixed size neighborhood of a geodesic segment $\left[u_{i} v_{i}\right]$ from $u_{i}$ to $v_{i}$ is contained in $\Omega$, while under the hypotheses of Theorem 4.3 the geodesic segment $\left[u_{i} v_{i}\right]$ is contained in $\Omega$ by convexity. It follows that, in both situations (and assuming the random walk in nearest-neighbor in the second situation), one has $G_{r}\left(u_{i}, v_{i} ; \Omega\right) \geq p_{\min }^{d\left(u_{i}, v_{i}\right)}$ for all $r \geq 1$, for some $p_{\min }>0$. Consequently,

$$
H\left(u_{i}, v_{i} ; B_{i+1}^{c}\right) \leq \lambda_{i} G_{r}\left(u_{i}, v_{i} ; \Omega\right),
$$

where $\lambda_{i}$ is superexponentially small in terms of $m(3 / 4)^{i}$. The $j$ th term of the first sum in (23) is therefore bounded by

$$
\lambda_{j} \sum_{\substack{u_{1}, \ldots, u_{j} \\ v_{1}, \ldots, v_{j}}} G_{r}\left(u_{j}, v_{j} ; \Omega\right) \prod_{i=0}^{j-1}\left\{H\left(u_{i}, u_{i+1} ; B_{i+1}^{c}\right) H\left(v_{i+1}, v_{i} ; B_{i+1}^{c}\right)\right\} .
$$

This matches the last line of (23) (with $j$ replacing $k$ ). Since every term in (23) is nonnegative, it follows that the $j$ th term of the first sum in (23) is bounded above by $\lambda_{j} G_{r}\left(u_{0}, v_{0} ; \Omega\right)$.

Now take $k=N$ (i.e., the last step of the construction), and consider the final sum in (23). Since the points $u_{N}$ and $v_{N}$ are within distance $A$ of $z$, we have $G_{r}\left(u_{N}, v_{N} ; \Omega\right) \leq$ $C_{A} G_{r}\left(u_{N}, z ; \Omega\right) G_{r}\left(z, v_{N} ; \Omega\right)$, for a suitable constant $C_{A}<\infty$. When this upper bound is substituted for the factor $G_{r}\left(u_{N}, v_{N} ; \Omega\right)$, the final sum factors (one factor involving only the $u_{i} \mathrm{~s}$, the other only the $v_{i} \mathrm{~s}$ ). Reversing the path decomposition (i.e., gluing trajectories instead of splitting them) shows that the two resulting factors are bounded respectively by $G_{r}\left(u_{0}, z ; \Omega\right)$ and $G_{r}\left(z, v_{0} ; \Omega\right)$. Thus, we have

$$
G_{r}\left(u_{0}, v_{0} ; \Omega\right) \leq\left(\sum_{j=0}^{N-1} \lambda_{j}\right) G_{r}\left(u_{0}, v_{0} ; \Omega\right)+C_{A} G_{r}\left(u_{0}, z ; \Omega\right) G_{r}\left(z, v_{0} ; \Omega\right) .
$$


If $A$ is large enough, the sum $\sum \lambda_{j}$ will be smaller than $\leq 1 / 2$. Hence, for all large $A$,

$$
G_{r}\left(u_{0}, v_{0} ; \Omega\right) \leq 2 C_{A} G_{r}\left(u_{0}, z ; \Omega\right) G_{r}\left(z, v_{0} ; \Omega\right) .
$$

\subsection{Hölder continuity of the Green's function}

In this paragraph, we explain how the controls on the Martin boundary given by Theorem 1.2 follow from the relative Ancona's inequalities of the previous paragraph. The strategy of the proof is essentially the same as that introduced by Anderson and Schoen [3] for a similar purpose in a slightly different setting, and the details follow [22] closely.

Definition 4.5. - Let $[x y]$ and $\left[x^{\prime} y^{\prime}\right]$ be geodesic segments in the Cayley graph of $\Gamma$, and let $\varepsilon>0$. We say that the segment $\left[x^{\prime} y^{\prime}\right]$ shadows (more precisely, $\varepsilon$-shadows) the segment $[x y]$ if every point of $[x y]$ lies within distance $\varepsilon$ of $\left[x^{\prime} y^{\prime}\right]$. If both $\left[x^{\prime \prime} y^{\prime \prime}\right]$ and $\left[x^{\prime} y^{\prime}\right](2 \delta)$-shadow $[x y]$, where $\delta$ is a Gromov constant for the Cayley graph, then we say that they are fellowtraveling along $[x y]$.

THEOREM 4.6. - There exist constants $C>0$ and $\varrho<1$ such that for any geodesic segment $\left[x_{0} y_{0}\right]$, if $[x y]$ and $\left[x^{\prime} y^{\prime}\right]$ are fellow-traveling along $\left[x_{0} y_{0}\right]$, then

$$
\left|\frac{G_{r}(x, y) / G_{r}\left(x^{\prime}, y\right)}{G_{r}\left(x, y^{\prime}\right) / G_{r}\left(x^{\prime}, y^{\prime}\right)}-1\right| \leq C \varrho^{k},
$$

for all $r \in[1, R]$, where $k$ is the length of $\left[x_{0} y_{0}\right]$.

A direct application of Ancona's inequalities imply that

$$
\frac{G_{r}(x, y)}{G_{r}\left(x^{\prime}, y\right)} \asymp \frac{G_{r}\left(x, x_{0}\right)}{G_{r}\left(x^{\prime}, x_{0}\right)} \asymp \frac{G_{r}\left(x, y^{\prime}\right)}{G_{r}\left(x^{\prime}, y^{\prime}\right)} .
$$

The theorem is a quantitative strengthening of this estimate, showing that the ratio between those quantities not only stays bounded, but tends exponentially fast to 1 when $k$ tends to infinity.

In particular, take $x^{\prime}=1$ and let $y_{n}, y_{m}$ be points at distances $n, m$ from $x^{\prime}$ along a geodesic ray converging towards a point $\zeta \in \partial \Gamma$. For any fixed $x \in \Gamma$ the geodesic segments from $x$ or $x^{\prime}$ to $y_{n}$ or $y_{m}$ are fellow traveling along a geodesic segment of length at least $\min (m, n)-2 d\left(x, x^{\prime}\right)$. Therefore,

$$
\left|\frac{G_{r}\left(x, y_{n}\right) / G_{r}\left(1, y_{n}\right)}{G_{r}\left(x, y_{m}\right) / G_{r}\left(1, y_{m}\right)}-1\right| \leq C_{x} \varrho^{\min (m, n)}
$$

for a constant $C_{x}<\infty$ depending on $x$. This shows that the sequence $G_{r}\left(x, y_{n}\right) / G_{r}\left(1, y_{n}\right)$ is Cauchy, therefore convergent to a limit $K_{r}(x, \zeta)$. To prove Theorem 1.1, one should additionally show that the functions $K_{r}(x, \zeta)$ are minimal, and that for $\zeta \neq \zeta^{\prime}$ one has $K_{r}(x, \zeta) \neq K_{r}\left(x, \zeta^{\prime}\right)$. Ancona proved this for $r<R$ in [1], and his proofs also work for $r=R$ once the Ancona inequalities are established. Finally, letting $m$ tend to infinity, one gets $\left|G_{r}\left(x, y_{n}\right) / G_{r}\left(1, y_{n}\right)-K_{r}(x, \zeta)\right| \leq C_{x} \varrho^{n} K_{r}(x, \zeta)$, which proves Theorem 1.2. 
Proof of Theorem 4.6. - Let $L \gg 2 \delta$ be a large constant, and consider a geodesic segment $\left[x_{0} y_{0}\right]$ of length $k \gg L$. For each $1 \leq i \leq \ell:=k /(3 L)$, define $\Omega_{i}$ to be the set of all $z \in \Gamma$ such that every geodesic segment from $y_{0}$ to $z$ passes through the ball of radius $2 \delta$ centered at the point $z_{i}$ at distance $3 L i$ from $y_{0}$ along $\left[x_{0} y_{0}\right]$. The sequence of domains $\Omega_{i}$ is decreasing in $i$ (by an easy application of the thin triangle property). Moreover, if the geodesic segments $[x y]$ and $\left[x^{\prime} y^{\prime}\right]$ are fellow-traveling along $\left[x_{0} y_{0}\right]$, then both $x, x^{\prime} \in \Omega_{\ell}$ and $y, y^{\prime} \in \Omega_{1}^{c}$.

Consider the function $u(z)=G_{r}(z, y) / G_{r}\left(x_{0}, y\right)$, which is $r$-harmonic on $\Omega_{1}$ and normalized by $u\left(x_{0}\right)=1$. Starting with $u_{1}=u$, we will inductively construct a sequence of $r$-harmonic functions $u_{i}$ on $\Omega_{i}$, with $u_{i-1}=u_{i}+\varphi_{i}$, in such a way that $\varphi_{i}$ does not depend on the initial normalized harmonic function $u$, and so that $u_{i-1} \geq \varphi_{i} \geq \varepsilon u_{i-1}$ on $\Omega_{i}$, for some $\varepsilon>0$. Let us first show how this gives the conclusion of the theorem.

As $\varphi_{i} \geq \varepsilon u_{i-1}$, we have $u_{i} \leq(1-\varepsilon) u_{i-1}$, hence $u_{\ell} \leq(1-\varepsilon)^{\ell-1} u_{1}$. Applying the same construction to $v(z)=G_{r}\left(z, y^{\prime}\right) / G_{r}\left(x_{0}, y^{\prime}\right)$, we obtain $v=\sum \varphi_{i}+v_{\ell}$ (for the same functions $\left.\varphi_{i}\right)$, which gives on $\Omega_{\ell}$ the estimate $|u-v|=\left|u_{\ell}-v_{\ell}\right| \leq C(1-\varepsilon)^{\ell}(u+v)$. Since $u \asymp v$ by (24), we get $|u / v-1| \leq C(1-\varepsilon)^{\ell}$ on $\Omega_{\ell}$, which is the desired inequality.

We now describe the construction of $\varphi_{i}$. Assume that $u_{1}, \ldots, u_{i}$ have been defined. By harmonicity, for any $z \in \Omega_{i+1}$,

$$
u_{i}(z)=\sum_{w \in \Omega_{i}^{c}} G_{r}\left(z, w ; \Omega_{i}\right) u_{i}(w) .
$$

Define $\Lambda_{i}$ to be the set of all $z \in \Omega_{i}$ such that $\left(x_{0} \mid z\right)_{y_{0}} \in\left[3 L i+L-C_{0}, 3 L i+L+C_{0}\right]$, where $(x \mid y)_{z}=(d(x, z)+d(y, z)-d(x, y)) / 2$ is the Gromov product, which measures the distance along which two geodesic segments from $z$ to $x$ and from $z$ to $y$ are fellow traveling. This set is contained in $\Omega_{i}$, but is bounded away from $\Omega_{i+1}$, and any trajectory from $\Omega_{i+1}$ to the complement of $\Omega_{i}$ has to cross $\Lambda_{i}$. Splitting a trajectory from $z \in \Omega_{i+1}$ to $w \in \Omega_{i}^{c}$ according to its last visit to $\Lambda_{i}$, we get $G_{r}\left(z, w ; \Omega_{i}\right)=\sum_{w^{\prime} \in \Lambda_{i}} G_{r}\left(z, w^{\prime} ; \Omega_{i}\right) G_{r}\left(w^{\prime}, w ; \Omega_{i} \cap \Lambda_{i}^{c}\right)$.

We are now in a position to estimate $G_{r}\left(z, w^{\prime} ; \Omega_{i}\right)$ using Ancona's inequalities. Indeed, the geodesic segment from $z$ to $w^{\prime}$ passes within $2 \delta$ of the point $z_{i}^{*}$ at distance $3 L i+2 L$ from $y_{0}$ on $\left[x_{0} y_{0}\right]$, by hyperbolicity. Moreover, the domain $\Omega_{i}$ satisfies the assumptions of Theorem 4.1 (this readily follows from a tree approximation). Hence, $G_{r}\left(z, w^{\prime} ; \Omega_{i}\right) \leq C G_{r}\left(z, z_{i}^{*} ; \Omega_{i}\right) G_{r}\left(z_{i}^{*}, w^{\prime} ; \Omega_{i}\right)$, and so

$$
\begin{aligned}
u_{i}(z) & =\sum_{w \in \Omega_{i}^{c}} \sum_{w^{\prime} \in \Lambda_{i}} G_{r}\left(z, w^{\prime} ; \Omega_{i}\right) G_{r}\left(w^{\prime}, w ; \Omega_{i} \cap \Lambda_{i}^{c}\right) u_{i}(w) \\
& \leq C G_{r}\left(z, z_{i}^{*} ; \Omega_{i}\right) \sum_{w \in \Omega_{i}^{c}} \sum_{w^{\prime} \in \Lambda_{i}} G_{r}\left(z_{i}^{*}, w^{\prime} ; \Omega_{i}\right) G_{r}\left(w^{\prime}, w ; \Omega_{i} \cap \Lambda_{i}^{c}\right) u_{i}(w) \\
& =C G_{r}\left(z, z_{i}^{*} ; \Omega_{i}\right) u_{i}\left(z_{i}^{*}\right) .
\end{aligned}
$$

Replacing Ancona's inequality by the trivial bound

$$
G_{r}\left(z, w^{\prime} ; \Omega_{i}\right) \geq C^{\prime} G_{r}\left(z, z_{i}^{*} ; \Omega_{i}\right) G_{r}\left(z_{i}^{*}, w^{\prime} ; \Omega_{i}\right),
$$

we also get a lower bound in the last equation. Hence, $u_{i}(z) \asymp G_{r}\left(z, z_{i}^{*} ; \Omega_{i}\right) u_{i}\left(z_{i}^{*}\right)$ on $\Omega_{i+1}$. Using this estimate for $z=x_{0}$, we obtain $u_{i}(z) / u_{i}\left(x_{0}\right) \asymp G_{r}\left(z, z_{i}^{*} ; \Omega_{i}\right) / G_{r}\left(x_{0}, z_{i}^{*} ; \Omega_{i}\right)$. In particular, if $c$ is small enough, the function $\varphi_{i+1}(z)=c u_{i}\left(x_{0}\right) G_{r}\left(z, z_{i}^{*} ; \Omega_{i}\right) / G_{r}\left(x_{0}, z_{i}^{*} ; \Omega_{i}\right)$ satisfies $\varepsilon u_{i} \leq \varphi_{i+1} \leq u_{i}$ on $\Omega_{i+1}$. Moreover, this function only depends on $u_{i}$ through the 
value of $u_{i}\left(x_{0}\right)$. By induction, it only depends on $u\left(x_{0}\right)$. Since we have normalized $u$ so that $u\left(x_{0}\right)=1$, this shows that $\varphi_{i+1}$ is independent of the initial function $u$.

\section{Automatic structure}

\subsection{Strongly Markov groups and hyperbolicity}

A finitely generated group $\Gamma$ is said to be strongly Markov (fortement Markov—see [19]) if for each finite, symmetric generating set $A$ there exists a finite directed graph $\mathscr{C}=\left(V, E, s_{*}\right)$ with distinguished vertex $s_{*}$ ("start") and a labeling $\alpha: E \rightarrow A$ of edges by generators that meets the following specifications. A path in the graph is a sequence of edges $e_{0}, \ldots, e_{m-1}$ such that the endpoint of $e_{i}$ is the starting point of $e_{i+1}$. Let

$$
\mathscr{P}:=\left\{\text { finite paths in } \mathscr{G} \text { starting at } s_{*}\right\},
$$

and for each path $\gamma=e_{0} e_{1} \cdots e_{m-1}$, denote by

$$
\begin{aligned}
\alpha(\gamma) & =\text { path in } G^{\Gamma} \text { through } 1, \alpha\left(e_{0}\right), \alpha\left(e_{0}\right) \alpha\left(e_{1}\right), \ldots, \quad \text { and } \\
\alpha_{*}(\gamma) & =\alpha\left(e_{0}\right) \alpha\left(e_{1}\right) \cdots \alpha\left(e_{m-1}\right), \text { the right endpoint of } \alpha(\gamma) .
\end{aligned}
$$

Definition 5.1. - The labeled automaton $(\mathscr{G}, \alpha)$ is a strongly Markov automatic structure for $\Gamma$ if:

(A) No edge $e \in E$ ends at $s_{*}$.

(B) Every vertex $v \in V$ is accessible from the start state $s_{*}$.

(C) For every path $\gamma$, the path $\alpha(\gamma)$ is a geodesic path in $G^{\Gamma}$.

(D) The endpoint mapping $\alpha_{*}: \mathscr{P} \rightarrow \Gamma$ induced by $\alpha$ is a bijection of $\mathscr{P}$ onto $\Gamma$.

THEOREM 5.2. - Every word hyperbolic group is strongly Markov.

See [19], Ch. 9, Th. 13. The result is essentially due to Cannon (at least in a more restricted form) — see [14], [13] — and in important special cases (co-compact Fuchsian groups) to Series [34]. Henceforth, we will call the directed graph $\mathscr{Q}=\left(V, E, s_{*}\right)$ the Cannon automaton (despite the fact that it is not quite the same automaton as constructed in [14]).

Properties (C)-(D) of Definition 5.1 imply that for each $x \in \Gamma$ there is a unique geodesic segment in the Cayley graph from the group identity 1 to $x$ that is the image of a path in the automaton. We shall denote this distinguished geodesic segment by $L(1, x)$.

\subsection{Recurrent and transient vertices}

Let $\mathscr{C}$ be a Cannon automaton for the group $\Gamma$ with vertex set $V$ and (directed) edge set $E$. Call an edge $e \in E$ recurrent if there is a path in $\mathscr{G}$ of length $\geq 2$ that begins and ends with $e$; otherwise, call it transient. Denote by $\mathscr{C}_{R}$ the restriction of the digraph $\mathscr{C}$ to the set $\mathscr{R}$ of recurrent edges. For certain hyperbolic groups - among them the co-compact Fuchsian groups - the automatic structure can be chosen so that the digraph $\mathscr{C}_{R}$ is strongly connected (see [34]), i.e., for any two recurrent edges $e$ and $e^{\prime}$ there is a path from $e$ to $e^{\prime}$. Henceforth we restrict attention to word-hyperbolic groups with this property:

Assumption 5.3. - The automatic structure can be chosen so that the digraph $\mathscr{G}_{R}$ is strongly connected.

$4^{\text {e }}$ SÉRIE - TOME $46-2013$ - No 1 
Assumption 5.4. - The incidence matrix of the digraph $\mathscr{G}_{R}$ is aperiodic.

Both assumptions hold for any co-compact Fuchsian group. Assumption 5.4 is for ease of exposition only - the results and arguments below can be modified to account for any periodicities that might arise if the assumption were to fail. Assumption 5.3, however, is essentially important.

\subsection{Symbolic dynamics}

We shall assume for the remainder of the paper that the automaton $\mathscr{C}$ has been chosen so as to satisfy Assumptions 5.3 and 5.4.

Set

$$
\begin{aligned}
\Sigma & =\{\text { semi-infinite paths in } \mathscr{G}\} \\
\Sigma^{n} & =\{\text { paths of length } n \text { in } \mathscr{C}\} \\
\Sigma^{*} & =\cup_{n=0}^{\infty} \Sigma^{n} \\
\bar{\Sigma} & =\Sigma \cup \Sigma^{*} .
\end{aligned}
$$

By convention, there is a single path of length 0 , the empty path, that we denote by $\varnothing$. In some circumstances, it is useful to identify finite paths with semi-infinite paths in an automaton with an additional "cemetery" state, or with doubly-infinite paths in an automaton with two additional states ("embryo" and "cemetery"). This point of view makes it possible to apply directly in our setting results that are formulated in the literature only for semi-infinite paths. However, we stick to the notation with finite paths since it makes the correspondence with geodesic segments in the group (see below) more transparent.

We will also need bilateral versions of these sets, that we will denote with a subscript $\mathbb{Z}$. For instance, $\bar{\Sigma}_{\mathbb{Z}}$ is the set of (finite or infinite) bilateral paths in $\mathscr{C}$. Equivalently, it is the set of sequences $\left(\omega_{n}\right)_{n \in \mathbb{Z}}$ where $\omega_{n}$ is an edge of $\mathscr{C}$ for $n$ in some interval of $\mathbb{Z}$ (with admissible transition from $\omega_{n}$ to $\omega_{n+1}$ ), and $\omega_{n}$ is empty for $n$ outside of this interval. Let $\sigma$ be the forward shift operator on $\bar{\Sigma}$ and $\bar{\Sigma}_{\mathbb{Z}}$. The spaces $\bar{\Sigma}$ and $\bar{\Sigma}_{\mathbb{Z}}$ are given metrics in the usual way, that is,

$$
d\left(\omega, \omega^{\prime}\right)=2^{-n\left(\omega, \omega^{\prime}\right)}
$$

where $n\left(\omega, \omega^{\prime}\right)$ is the maximum integer $n$ such that $\omega_{i}=\omega_{i}^{\prime}$ for all $|i|<n$. With the topology induced by $d$ the space $\Sigma$ is a Cantor set, $\Sigma$ is the set of accumulation points of $\Sigma^{*}$, and $\bar{\Sigma}$ and $\bar{\Sigma}_{\mathbb{Z}}$ are compact. Observe that, relative to the metrics $d$, Hölder-continuous, real-valued functions on $\Sigma^{*}$ extend by continuity to Hölder-continuous functions on $\bar{\Sigma}$, and then pull back to Hölder-continuous functions on $\bar{\Sigma}_{\mathbb{Z}}$.

Each $\omega \in \Sigma$ projects via the edge-labeling map $\alpha$ to a geodesic ray in $G^{\Gamma}$ starting at the vertex 1 (more precisely, the sequence of finite prefixes of $\omega$ project to the vertices along a geodesic ray). Each geodesic ray in $G^{\Gamma}$ must converge in the Gromov topology to a point of $\partial \Gamma$, so $\alpha$ induces on $\Sigma$ a mapping $\alpha_{*}$ to $\partial \Gamma$. By construction, this mapping is Hölder continuous relative to any visual metric on $\partial \Gamma$. Moreover, because each $\zeta \in \partial \Gamma$ is the limit of a geodesic ray starting at the vertex 1 , the induced mapping $\alpha_{*}$ is surjective.

In a somewhat different way, the edge-labeling map $\alpha$ determines a map from the space $\bar{\Sigma}_{\mathbb{Z}}$ to the set of two-sided (finite or infinite) geodesics in $G^{\Gamma}$ that pass through the vertex 1. 
This map is defined as follows: if $\omega \in \bar{\Sigma}_{\mathbb{Z}}$ then the image of $\omega$ is the two-sided geodesic that passes through

$$
\ldots, \alpha\left(\omega_{-1}^{-1}\right) \alpha\left(\omega_{-2}^{-1}\right), \alpha\left(\omega_{-1}^{-1}\right), 1, \alpha\left(\omega_{0}\right), \alpha\left(\omega_{0}\right) \alpha\left(\omega_{1}\right), \ldots,
$$

equivalently, it is the concatenation of the geodesic rays starting at 1 that are obtained by reading successive steps from the sequences

$$
\omega_{0} \omega_{1} \omega_{2} \cdots \text { and } \omega_{-1}^{-1} \omega_{-2}^{-1} \omega_{-3}^{-1} \cdots,
$$

respectively. When $\omega$ is bi-infinite, each of these geodesic rays converges to a point of $\partial \Gamma$, so $\alpha$ induces a mapping from $\Sigma_{\mathbb{Z}}$ into $\partial \Gamma \times \partial \Gamma$. This mapping is neither injective nor surjective, but it is Hölder-continuous.

Let $E_{*}$ be the set of edges originating from $s_{*}$, and let $\Sigma^{m}\left(E_{*}\right)$ be the set of sequences of length $m$ in $\Sigma^{m}$ with $\omega_{0} \in E_{*}$. By definition of the Cannon automaton, the mapping $\alpha_{*}$ induces a bijection between $\Sigma^{m}\left(E_{*}\right)$ and the sphere $S_{m}$ of radius $m$ in $G^{\Gamma}$.

COROLlary 5.5. - Let $\zeta$ be the spectral radius of the incidence matrix of the digraph $\mathscr{Q}$. If Assumptions 5.3 and 5.4 hold, then $\zeta>1$, and there exists $C>0$ such that

$$
\left|S_{m}\right| \sim C \zeta^{m} \text { as } m \rightarrow \infty .
$$

Proof. - This follows directly from the Perron-Frobenius theorem, with the exception of the assertion that the spectral radius $\zeta>1$. That $\zeta>1$ follows from the fact that the group $\Gamma$ is nonelementary. Since $\Gamma$ is nonelementary, it is nonamenable, and so its Cayley graph has positive Cheeger constant; this implies that $\left|S_{m}\right|$ grows exponentially with $m$.

COROLlary 5.6. - The shift $(\Sigma, \sigma)$ has positive topological entropy.

Proof. - This follows from the exponential growth of the group, cf. Corollary 5.5.

\section{Thermodynamic formalism}

Assume throughout this section and Sections 7-8 that the group $\Gamma$ is co-compact Fuchsian, and that the random walk is symmetric and finite-range.

\subsection{The potential functions $\varphi_{r}$}

The machinery of thermodynamic formalism and Gibbs states developed in [11] applies to Hölder continuous functions on $\Sigma$ (or on $\bar{\Sigma}$ ). To make use of this machinery, we will lift the Green's function and the Martin kernel from $\bar{\Gamma}$ to the sequence space $\bar{\Sigma}$. For this the results of Theorem 1.2 and Theorem 4.6 are crucial, as they ensure that those lifts are Höldercontinuous. The lift is defined as follows. For $\omega \in \Sigma^{*}$, set

$$
\varphi_{r}(\omega):=\log \frac{G_{r}\left(1, \alpha_{*}(\omega)\right)}{G_{r}\left(1, \alpha_{*}(\sigma \omega)\right)} .
$$

If $\omega$ is not the empty path, one can also write

$$
\varphi_{r}(\omega)=\log \frac{G_{r}\left(1, \alpha_{*}(\omega)\right)}{G_{r}\left(\alpha_{*}\left(\omega_{0}\right), \alpha_{*}(\omega)\right)} .
$$

Therefore, Theorem 4.6 shows that, if two paths $\omega$ and $\omega^{\prime}$ coincide up to time $n$, then $\left|\varphi_{r}(\omega)-\varphi_{r}\left(\omega^{\prime}\right)\right| \leq C \varrho^{n}$, for some $\varrho<1$. By definition of the distance on $\Sigma^{*}$, this means 
that $\varphi_{r}$ is Hölder-continuous. In particular, it extends to a Hölder-continuous function (that we still denote by $\varphi_{r}$ ) on $\bar{\Sigma}$. On $\Sigma$, it is given by

$$
\varphi_{r}(\omega)=\log \frac{K_{r}\left(1, \alpha_{*}(\omega)\right)}{K_{r}\left(\alpha_{*}\left(\omega_{0}\right), \alpha_{*}(\omega)\right)}=-\log K_{r}\left(\alpha_{*}\left(\omega_{0}\right), \alpha_{*}(\omega)\right) .
$$

The mapping $r \mapsto \varphi_{r}$ is clearly continuous at every point of $\Sigma^{*}$, and all the functions $\varphi_{r}$ are uniformly Hölder-continuous for some fixed exponent. Therefore, $r \mapsto \varphi_{r}$ is also continuous for the sup norm, and it follows that it is continuous for the Hölder topology respective to any Hölder exponent strictly less than the initial one.

By construction, if $\omega$ is of length $n$,

$$
G_{r}\left(1, \alpha_{*}(\omega)\right)=G_{r}(1,1) \exp \left(S_{n} \varphi_{r}(\omega)\right)
$$

where (in Bowen's notation [11])

$$
S_{n} \varphi:=\sum_{j=0}^{n-1} \varphi \circ \sigma^{j} .
$$

(Unfortunately, the notation $S_{n} \varphi$ conflicts with the notation $S_{m}$ for the sphere of radius $m$ in $\Gamma$; however, both notations are standard, and the meaning should be clear in the following by context.)

\subsection{Gibbs states: background}

According to a fundamental theorem of ergodic theory (cf. [11], Th. 1.2 and sec. 1.4), for each Hölder continuous function on a topologically mixing subshift of finite type, there is a unique Gibbs state for this potential. Unfortunately, the subshift of finite type induced by a Cannon automaton is not topologically mixing, since the edges originating from $s_{*}$ are always transient (and for hyperbolic groups in general, there can also be terminal edges, i.e., edges where a path cannot be continued). However, under Assumptions 5.3 and 5.4, the recurrent part of the graph is topologically mixing. Therefore, the existence of Gibbs states and the corresponding Ruelle operator theory will generalize to our setting.

Consider a finite directed graph whose recurrent part is connected and aperiodic, let $\bar{\Sigma}$ be the set of finite or infinite paths in this graph, and let $\sigma: \bar{\Sigma} \rightarrow \bar{\Sigma}$ be the left shift. The assumption that the recurrent part of the graph is connected and aperiodic implies that the restriction of $\sigma$ to the set of infinite paths in the recurrent set is topologically mixing. Let $\mathcal{H}$ be the space of real-valued Hölder-continuous functions on $\bar{\Sigma}$ (for some fixed Hölder exponent). Let $\mathscr{R}$ be the set of recurrent edges in the graph, $\mathscr{R}^{+}$the set of edges that can be reached from a recurrent edge, and $\mathscr{R}^{-}$the set of edges from which a recurrent edge can be reached.

Theorem 6.1. - For any potential $\varphi \in \mathcal{H}$, define an operator $\mathscr{L}_{\varphi}$ acting on continuous functions $f: \bar{\Sigma} \rightarrow \mathbb{R}$ by

$$
\mathscr{L}_{\varphi} f(\omega)=\sum_{\sigma\left(\omega^{\prime}\right)=\omega} e^{\varphi\left(\omega^{\prime}\right)} f\left(\omega^{\prime}\right)
$$

where if $\omega$ is the empty path the sum is restricted to the preimages $\omega^{\prime}$ of positive length. There exist a real number $\operatorname{Pr}(\varphi)$ (the pressure of $\varphi$ ), a number $\varepsilon>0$, a Hölder-continuous function 
$h_{\varphi}: \bar{\Sigma} \rightarrow \mathbb{R}^{+}$and a probability measure $\nu_{\varphi}$ on $\bar{\Sigma}$ such that, for any $f \in \mathcal{H}$, the following asymptotics hold in $\mathcal{H}$ :

$$
\mathscr{L}_{\varphi}^{n} f=e^{n \operatorname{Pr}(\varphi)}\left(\int f d \nu_{\varphi}\right) h_{\varphi}+O\left(e^{-\varepsilon n} e^{n \operatorname{Pr}(\varphi)}\right) .
$$

The support of the function $h_{\varphi}$ is the set of sequences whose elements all belong to $\mathcal{R}^{+}$, and $h_{\varphi}$ is bounded away from zero there. The support of the measure $\nu_{\varphi}$ is the set of infinite sequences whose elements all belong to $\mathscr{R}^{-}$.

The measure $\mu_{\varphi}=h_{\varphi} \nu_{\varphi}$ is the Gibbs measure associated to the potential $\varphi$ : it is a $\sigma$-invariant probability measure supported by the recurrent part $\Sigma^{\mathscr{R}}$ of $\Sigma$, and it satisfies, for any $\omega=\left(\omega_{n}\right) \in \Sigma^{\mathscr{R}}$,

$$
C_{1} \leq \frac{\mu_{\varphi}\left[\omega_{0}, \ldots, \omega_{n-1}\right]}{e^{S_{n} \varphi(\omega)-n \operatorname{Pr}(\varphi)}} \leq C_{2}
$$

where $C_{1}, C_{2}>0$ are two constants and the cylinder $\left[\omega_{0}, \ldots, \omega_{n-1}\right]$ is the set of sequences $\omega^{\prime}=\left(\omega_{0}^{\prime}, \omega_{1}^{\prime}, \ldots\right)$ with $\omega_{i}^{\prime}=\omega_{i}$ for $0 \leq i \leq n-1$.

Finally, all the quantities in the statement of the theorem (i.e., $\operatorname{Pr}(\varphi), \varepsilon, h_{\varphi}, \nu_{\varphi}, C_{1}, C_{2}, \mu_{\varphi}$ and the implicit constant in the $O$-term in (29)) vary continuously with $\varphi \in \mathcal{H}$.

When the subshift of finite type is topologically mixing, this theorem is proved in [11]. Since the arguments there are easily adapted to obtain the above version, we will only sketch a proof, emphasizing the arguments that differ from those of [11].

Proof. - Standard arguments using Lasota-Yorke estimates (see for instance [32] or [5]) show that $\mathscr{L}_{\varphi}$ has a spectral gap on $\mathcal{H}$ : denoting by $e^{\operatorname{Pr}(\varphi)}$ the spectral radius of $\mathscr{L}_{\varphi}$, this operator has finitely many eigenvalues of modulus $e^{\operatorname{Pr}(\varphi)}$, and the rest of its spectrum is contained in a disk of strictly smaller radius. Using the positivity of $e^{\varphi}$ and the fact that the recurrent part of $\Sigma$ is topologically mixing, one can then prove that there is a unique eigenvalue of maximal modulus, and that it is simple. The asymptotics (29) follows. The eigenfunction and eigenmeasure $h_{\varphi}$ and $\nu_{\varphi}$ satisfy respectively $\mathscr{L}_{\varphi} h_{\varphi}=e^{\operatorname{Pr}(\varphi)} h_{\varphi}$ and $\mathcal{L}_{\varphi}^{*} \nu_{\varphi}=e^{\operatorname{Pr}(\varphi)} \nu_{\varphi}$.

Consider next the support of $h_{\varphi}$. The results in [11] imply that $h_{\varphi}$ is positive, and bounded from below, on the recurrent part $\Sigma^{\mathscr{R}}$ of $\Sigma$. Since $h_{\varphi}$ is Hölder continuous, this implies that, if $n$ is large enough, then $h_{\varphi}$ is also positive on elements of $\bar{\Sigma}$ of length at least $n$ whose first $n$ symbols are in $\mathscr{R}$. Consider now a sequence $\omega$ whose symbols all belong to $\mathcal{R}^{+}$. There exists a sequence $\alpha$ beginning by $n$ symbols in $\mathscr{R}$ such that $\alpha \omega$ is a possible path in the automaton. Therefore,

$$
h_{\varphi}(\omega)=e^{-n \operatorname{Pr}(\varphi)} \sum_{\sigma^{n}(\eta)=\omega} e^{S_{n} \varphi(\eta)} h_{\varphi}(\eta) \geq e^{-n \operatorname{Pr}(\varphi)} e^{S_{n} \varphi(\alpha \omega)} h_{\varphi}(\alpha \omega)>0 .
$$

On the other hand, if $\omega$ contains a symbol not belonging to $\mathcal{R}^{+}$, then $\omega$ has no preimage under $\sigma^{n}$ if there is no path of length $n$ in the transient part of the automaton. It follows that $\mathscr{L}_{\varphi}^{n} h_{\varphi}(\omega)=0$, hence $h_{\varphi}(\omega)=0$. This shows that the support of $h_{\varphi}$ is exactly those sequences with all symbols in $\mathscr{R}^{+}$. Since this set is compact, $h_{\varphi}$ is bounded from below there.

If $f$ is a continuous function, then

$$
\nu_{\varphi}(f)=e^{-n \operatorname{Pr}(\varphi)} \nu_{\varphi}\left(\mathscr{L}_{\varphi}^{n} f\right) .
$$

4 e SÉRIE - TOME $46-2013-$ N $^{\circ} 1$ 
Since $\mathcal{L}_{\varphi}^{n} f$ only depends on the values of $f$ on paths of length at least $n$, this shows that $\nu_{\varphi}$ has no atom on paths of finite length. Let us now take $f=1_{[C]}$ the characteristic function of a cylinder $[C]$ of length $n$. Since $\mathcal{L}_{\varphi}^{n} 1_{[C]}(\omega)=e^{S_{n} \phi(C \omega)}$ if the concatenation $C \omega$ is an admissible sequence, and 0 otherwise, we deduce that $\nu_{\varphi}[C]=0$ if $C$ can not be extended. If a cylinder contains a symbol not in $\mathscr{R}^{-}$, it is a union of cylinders that can not be extended, and has therefore 0 measure. On the other hand, if $C$ only contains symbols in $\mathscr{R}^{-}$, then $[C]$ contains a cylinder $\left[C^{\prime}\right]$ of some length $m$ that can be followed by a symbol $\omega_{0}$ in $\mathscr{R}$. Since $\mathcal{L}^{m} 1_{\left[C^{\prime}\right]}$ is bounded from below on $\left[\omega_{0}\right]$, we get $\nu_{\varphi}[C] \geq c \nu_{\varphi}\left[\omega_{0}\right]$, which is nonzero since $\nu_{\varphi}$ has full support in the recurrent part of $\Sigma$, by [11]. This shows that the support of $\nu_{\varphi}$ is exactly the set of infinite paths whose symbols all belong to $\mathcal{R}^{-}$.

The claims on the supports of $h_{\varphi}$ and $\nu_{\varphi}$ show that the probability measure $\mu_{\varphi}=h_{\varphi} \nu_{\varphi}$ is supported on the recurrent part of $\Sigma$. It coincides there with the Gibbs measure constructed in [11]. Hence, (30) follows.

Finally, all the quantities in the statement of the theorem are constructed from the spectral theory of the operator $\mathcal{L}_{\varphi}$. It then follows by standard arguments in regular perturbation theory that they all vary continuously with $\varphi$ in the Hölder topology.

\subsection{Gibbs states and Green's function on spheres}

Henceforth we denote by $\mu_{r}$ the Gibbs measure on $\bar{\Sigma}$ corresponding to the potential $\varphi_{r}$ defined by Equations (26) and (27). Let $\lambda_{r, m}$ be the probability measure on the sphere $S_{m} \subset \Gamma$ with density proportional to $G_{r}(1, x)^{2}$, that is, such that

$$
\lambda_{r, m}(x)=\frac{G_{r}(1, x)^{2}}{\sum_{y \in S_{m}} G_{r}(1, y)^{2}} \quad \text { for all } x \in S_{m} .
$$

Recall that $S_{m}$ is in one-to-one correspondence with the paths of length $m$ in the automaton $\mathscr{Q}$ that begin at $s_{*}$; in particular, each $x \in S_{m}$ corresponds uniquely to a path $\omega$ of length $m$ whose first step $\omega_{0}$ belongs to the set $E_{*}$ of edges originating from $s_{*}$. Thus, for each $m \geq 1$, the probability measure $\lambda_{r, m}$ on $S_{m}$ pulls back to a probability measure on $\Sigma^{m}\left(E_{*}\right) \subset \bar{\Sigma}$, which we also denote by $\lambda_{r, m}$. This measure has density proportional to

$$
G_{r}\left(1, \alpha_{*}(\omega)\right)^{2}=G_{r}(1,1)^{2} \exp \left\{2 S_{m} \varphi_{r}(\omega)\right\}
$$

where $\omega \in \Sigma^{m}\left(E_{*}\right)$.

Proposition 6.2. - For each $r \in[1, R]$, the measures $\lambda_{r, m}$ on $\bar{\Sigma}$ converge weakly as $m \rightarrow \infty$ to a probability measure $\lambda_{r}$ on $\bar{\Sigma}$, and this convergence holds uniformly in $r$, in the following sense: if $f: \bar{\Sigma} \rightarrow \mathbb{R}$ is continuous, then

$$
\lim _{m \rightarrow \infty} \int f d \lambda_{r, m}=\int f d \lambda_{r},
$$

uniformly for $r \in[1, R]$. Furthermore, there exist constants $0<C=C(r ; 2)<\infty$ (depending continuously on $r$ ) such that the normalizing constants in (31) satisfy

$$
\sum_{x \in S_{m}} G_{r}(1, x)^{2} \sim C \exp \left\{m \operatorname{Pr}\left(2 \varphi_{r}\right)\right\}
$$

as $m \rightarrow \infty$. 
Proof. - We first prove (33). Let $\mathcal{L}_{r}$ be the Ruelle operator associated to the potential $2 \varphi_{r}$. Denoting by $\varnothing$ the path of length 0 in $\bar{\Sigma}$, and by $1_{E_{*}}: \bar{\Sigma} \rightarrow \mathbb{R}$ the function equal to 1 on paths originating from $s_{*}$ and 0 otherwise, we have

$$
\mathscr{L}_{r}^{m} 1_{E_{*}}(\varnothing)=\sum e^{2 S_{m} \varphi_{r}(\omega)}=\sum G_{r}\left(1, \alpha_{*}(\omega)\right)^{2} / G_{r}(1,1)^{2},
$$

where the sum is over all paths $\omega$ of length $m$ originating from $s_{*}$. Since $\alpha_{*}$ induces a bijection between such words and $S_{m}$, we get

$$
\sum_{x \in S_{m}} G_{r}(1, x)^{2}=G_{r}(1,1)^{2} \mathscr{L}_{r}^{m} 1_{E_{*}}(\varnothing)
$$

By the Ruelle-Perron-Frobenius Theorem 6.1, this is asymptotic to

$$
G_{r}(1,1)^{2} e^{m \operatorname{Pr}\left(2 \varphi_{r}\right)}\left(\int 1_{E_{*}} d \nu_{r}\right) h_{r}(\varnothing),
$$

where $\nu_{r}$ and $h_{r}$ are the eigenmeasure and eigenfunction of $\mathcal{L}_{r}$. Since $\left(\int 1_{E_{*}} d \nu_{r}\right) h_{r}(\varnothing)>0$ by Theorem 6.1, we obtain (33).

We now turn to $\lambda_{r, m}$. This quantity can also be expressed in terms of the transfer operator, as follows:

$$
\int f d \lambda_{r, m}=\mathscr{L}_{r}^{m}\left(1_{E_{*}} f\right)(\varnothing) / \mathcal{L}_{r}^{m}\left(1_{E_{*}}\right)(\varnothing) .
$$

It follows again from Theorem 6.1 that, if $f$ is Hölder-continuous, then $\int f d \lambda_{r, m}$ converges to $\int 1_{E_{*}} f d \nu_{r} / \int 1_{E_{*}} d \nu_{r}$. Moreover, the convergence is uniform for $r \in[1, R]$. If $f$ is merely continuous, it can be uniformly approximated by a Hölder-continuous function, and the same result follows. The limiting measure $\lambda_{r}$ is the normalized restriction of $\nu_{r}$ to the paths starting from $s_{*}$.

Note 6.3. - Virtually the same argument shows that for any $\theta \in \mathbb{R}$, as $m \rightarrow \infty$,

$$
\sum_{x \in S_{m}} G_{r}(1, x)^{\theta} \sim C \exp \left\{m \operatorname{Pr}\left(\theta \varphi_{r}\right)\right\} .
$$

The result (33) implies that $\operatorname{Pr}\left(2 \varphi_{r}\right)<0$ for all $r<R$ (see Lemma 7.2 below), and Note 1.7 implies that $\operatorname{Pr}\left(\varphi_{r}\right)>0$ for all $r \in(1, R]$. Since $\operatorname{Pr}\left(\theta \varphi_{r}\right)$ varies continuously with $\theta$, it follows that for each $r \in(1, R]$ there exists $\theta \in(1,2]$ such that $\operatorname{Pr}\left(\theta \varphi_{r}\right)=0$. It can also be shown that the convergence of the sums is uniform in $r$ for $r \in[1, R]$.

Proposition 6.4. - Let $g: \bar{\Sigma} \rightarrow \mathbb{R}$ be any Hölder-continuous function. Then for each $\delta>0$

$$
\lim _{m \rightarrow \infty} \lambda_{r, m}\left\{\omega \in \Sigma^{m}\left(E_{*}\right):\left|\frac{1}{m} \sum_{j=0}^{m-1} g \circ \sigma^{j}(\omega)-\int g d \mu_{r}\right|>\delta\right\}=0,
$$

and the convergence is uniform in $r \in[1, R]$.

Proof. - To prove the convergence (35) for any particular $r \in[1, R]$ it suffices, by Chebychev's inequality, to prove that the variance of the average converges to zero as 
$m \rightarrow \infty$. Replacing $g$ by $g-\int g d \mu_{r}$ and $\varphi_{r}$ by $\varphi_{r}-\operatorname{Pr}\left(\varphi_{r}\right)$, we may assume that $\int g d \mu_{r}=0$ and $\operatorname{Pr}\left(\varphi_{r}\right)=0$. We then have

$$
\int\left(\sum_{j=0}^{m-1} g \circ \sigma^{j}\right)^{2} d \lambda_{r, m}=\sum_{j=0}^{m-1} \int\left(g \circ \sigma^{j}\right)^{2} d \lambda_{r, m}+2 \sum_{j<k} \int g \circ \sigma^{j} \cdot g \circ \sigma^{k} d \lambda_{r, m} .
$$

The first sum is bounded by $m\|g\|_{\infty}^{2}$. In the second sum, for each $j<k$, we have, by (34),

$$
\begin{aligned}
\int g \circ \sigma^{j} \cdot g \circ \sigma^{k} d \lambda_{r, m} & =\mathscr{L}_{r}^{m}\left(1_{E_{*}} g \circ \sigma^{j} \cdot g \circ \sigma^{k}\right)(\varnothing) / \mathcal{L}_{r}^{m} 1_{E_{*}}(\varnothing) \\
& =\mathscr{L}_{r}^{m-k}\left(g \mathcal{L}_{r}^{k-j}\left(g \mathcal{L}_{r}^{j} 1_{E_{*}}\right)\right)(\varnothing) / \mathcal{L}_{r}^{m} 1_{E_{*}}(\varnothing) .
\end{aligned}
$$

(The second equation follows from the identity $\mathscr{L}(u \cdot v \circ \sigma)=\mathscr{L}(u) \cdot v$.) Theorem 6.1 implies that $\mathcal{L}_{r}^{n} 1_{E_{*}}=\nu_{r}\left[E_{*}\right] h_{r}+O\left(e^{-\varepsilon n}\right)$, so the denominator $\mathcal{L}_{r}^{m} 1_{E_{*}}(\varnothing)$ converges as $m \rightarrow \infty$ to a positive constant $\nu_{r}\left[E_{*}\right] h_{r}(\varnothing)$, and

$$
\begin{aligned}
\left.\mathscr{L}_{r}^{k-j}\left(g \mathcal{L}_{r}^{j} 1_{E_{*}}\right)\right)=\nu_{r}\left[E_{*}\right] \mathcal{L}_{r}^{k-j}\left(g h_{r}\right) & +O\left(e^{-\varepsilon j}\right) \\
& =\nu_{r}\left[E_{*}\right]\left(\int g h_{r} d \nu_{r}\right) h_{r}+O\left(e^{-\varepsilon(k-j)}\right)+O\left(e^{-\varepsilon j}\right) .
\end{aligned}
$$

Since $\int g h_{r} d \nu_{r}=\int g d \mu_{r}=0$, this is $O\left(e^{-\varepsilon(k-j)}\right)+O\left(e^{-\varepsilon j}\right)$. Summing over $j<k<m$, we obtain the bound

$$
C \sum_{j<k<m}\left(e^{-\varepsilon j}+e^{-\varepsilon(k-j)}\right) \leq C m .
$$

This shows that the variance of the sum $S_{m} g$ is $O(m)$ as $m \rightarrow \infty$, and so (35) follows.

Finally, all of the estimates obtained in the argument are uniform in $r$, since all spectral data coming from Theorem 6.1 are already uniform.

The ergodic average in (35) is expressed as an average over the orbit of a path in the Cannon automaton, but it readily translates to an equivalent statement for ergodic averages along the geodesic segment $L=L(1, x)$, as we now explain. Consider a function $f$, defined on the set of geodesic segments through 1 in the Cayley graph $G^{\Gamma}$. We say that it is Hölder continuous if $\left|f(L)-f\left(L^{\prime}\right)\right| \leq C \varrho^{n}$ for some $\varrho<1$ whenever two geodesics $L$ and $L^{\prime}$ coincide on the ball of radius $n$ around 1 . We put $f(L)=0$ if $L$ is a geodesic segment not containing 1 . We have defined in (25) a map $\alpha$ associating to a (finite) bilateral path in $\Sigma_{\mathbb{Z}}^{*}$ a geodesic segment in $G^{\Gamma}$. Therefore, $f \circ \alpha$ is a function on $\Sigma_{\mathbb{Z}}^{*}$. It is Hölder continuous, and extends to a Hölder continuous function on $\bar{\Sigma}_{\mathbb{Z}}$.

There is a natural reference measure on the bilateral shift $\bar{\Sigma}_{\mathbb{Z}}$ : since the Gibbs measure $\mu_{r}$ constructed on $\Sigma$ in Theorem 6.1 is shift-invariant, it extends to a measure (denoted $\mu_{r}^{\mathbb{Z}}$ ) on $\Sigma_{\mathbb{Z}}$.

COROLlary 6.5. - Let $f$ be a Hölder continuous function on the space of geodesic segments through 1 in $G^{\Gamma}$. Then for each $\delta>0$,

$$
\lim _{m \rightarrow \infty} \lambda_{r, m}\left\{x \in S_{m}:\left|m^{-1} \sum_{z \in L(1, x)} f\left(z^{-1} L(1, x)\right)-\int f \circ \alpha d \mu_{r}^{\mathbb{Z}}\right|>\delta\right\}=0,
$$

and the convergence is uniform in $r \in[1, R]$. 
Proof. - Let $\tilde{f}=f \circ \alpha$. If the function $\tilde{f}$ only depends on the positive coordinates of a symbolic sequence, this statement directly reduces to Corollary 6.4 since $S_{m}$ is in bijection with the set of paths of length $m$ starting from $s_{*}$. By a theorem of Livsits ([11], Lemma 1.6one may either check that the proof still applies in our setting allowing finite paths, or add cemeteries to reduce our situation to the classical setting) any Hölder continuous function $\tilde{f}$ on $\bar{\Sigma}_{\mathbb{Z}}$ is cohomologous to a Hölder continuous function $g$ that depends only on the forward coordinates, i.e., there exists a Hölder continuous function $u$ such that $g=\tilde{f}+u-u \circ \sigma$. Since it is equivalent to have (36) for $\tilde{f}$ or $\tilde{f}+u-u \circ \sigma$, the general case follows.

\section{Evaluation of the pressure at $r=R$}

Proposition 6.2 implies that the sums $\sum_{y \in S_{m}} G_{R}(1, y)^{2}$ grow or decay sharply exponentially at exponential rate $\operatorname{Pr}\left(2 \varphi_{R}\right)$. Consequently, to prove the relation (7) of Theorem 1.6 it suffices to prove that this rate is 0 .

Proposition 7.1. $-\operatorname{Pr}\left(2 \varphi_{R}\right)=0$.

The second assertion (8) of Theorem 1.6 also follows from Proposition 7.1, by the main result of [26]. (If it could be shown that the cocycle $\varphi_{R}$ defined by (27) above is nonlattice in the sense of [26], then the result (8) could be strengthened from $\asymp$ to $\sim$.)

The remainder of this section is devoted to the proof of Proposition 7.1. The first step, that $\operatorname{Pr}\left(2 \varphi_{R}\right) \leq 0$, is a consequence of the differential Equations (9). These imply the following.

Lemma 7.2. - For every $r<R$,

$$
\begin{aligned}
& \operatorname{Pr}\left(2 \varphi_{r}\right)<0, \quad \text { and so } \\
& \operatorname{Pr}\left(2 \varphi_{R}\right) \leq 0 .
\end{aligned}
$$

Proof. - For $r<R$ the Green's function $G_{r}(1,1)$ is analytic in $r$, so its derivative must be finite. Thus, by Proposition 1.9, the sum $\sum_{x \in \Gamma} G_{r}(1, x)^{2}$ is finite. (The last term $r^{-1} G_{r}(1,1)$ in Equation (9) remains bounded as $r \rightarrow R$ - because $G_{R}(1,1)<,\infty$.) Proposition 6.2 therefore implies that $\operatorname{Pr}\left(2 \varphi_{r}\right)$ must be negative. Since $\operatorname{Pr}(\varphi)$ varies continuously in $\varphi$, relative to the Hölder norm, (37) follows.

Proof of Proposition 7.1. - To complete the proof it suffices, by the preceding lemma, to show that $\operatorname{Pr}\left(2 \varphi_{R}\right)$ cannot be negative. In view of Proposition 6.2, this is equivalent to showing that $\sum_{x \in S_{m}} G_{R}(1, x)^{2}$ cannot decay exponentially in $m$. This will be accomplished by proving that exponential decay of $\sum_{x \in S_{m}} G_{R}(1, x)^{2}$ would force

$$
G_{r}(1,1)<\infty \text { for some } r>R,
$$

which is impossible since $R$ is the radius of convergence of the Green's function.

To prove (38), we will use the branching random walk interpretation of the Green's function discussed in Sec. 2.4. ${ }^{(2)}$ Recall that a branching random walk on the Cayley graph $G^{\Gamma}$ is specified by an offspring distribution $Q$; assume for definiteness that this is the Poisson distribution with mean $r>0$. At each step, particles first produce offspring particles

\footnotetext{
(2) Logically this is unnecessary - the argument has an equivalent formulation in terms of weighted paths, using (13) — but the branching random walk interpretation seems more natural. 
according to this distribution, independently, and then each of these particles jumps to a randomly chosen neighboring vertex. If the mean of the offspring distribution is $r>0$, and if the branching random walk is initiated by a single particle at the root 1 , then the mean number of particles located at vertex $x$ at time $n \geq 1$ is $r^{n} P^{1}\left\{X_{n}=x\right\}$. Thus, in particular, $G_{r}(1,1)$ equals the expected total number of particle visits to the root vertex 1 . The strategy is to show that if $\sum_{x \in S_{m}} G_{R}(1, x)^{2}$ decays exponentially in $m$, then for some $r>R$ the branching random walk remains subcritical, that is, the expected total number of particle visits to 1 is finite.

Recall that the Poisson distribution with mean $r>R$ is the convolution of Poisson distributions with means $R$ and $\varepsilon:=r-R$, that is, the result of adding independent random variables $U, V$ with distributions Poisson- $R$ and Poisson- $\varepsilon$ is a random variable $U+V$ with distribution Poisson- $r$. Thus, each reproduction step in the branching random walk can be done by making independent draws $U, V$ from the Poisson- $R$ and Poisson- $\varepsilon$ distributions. Use these independent draws to assign colors $k=0,1,2, \ldots$ to the particles according to the following rules:

(a) The ancestral particle at vertex 1 has color $k=0$.

(b) Any offspring resulting from a $U$-draw has the same color as its parent.

(c) Any offspring resulting from a $V$-draw has color equal to $1+$ the color of its parent.

Lemma 7.3. - For each $k=0,1,2, \ldots$, the expected number of visits to the vertex $y$ by particles of color $k$ is

$$
v_{k}(y)=\varepsilon^{k} \sum_{x_{1}, x_{2}, \ldots x_{k} \in \Gamma} G_{R}\left(1, x_{1}\right)\left(\prod_{i=1}^{k-1} G_{R}\left(x_{i}, x_{i+1}\right)\right) G_{R}\left(x_{k}, y\right) .
$$

Proof. - By induction on $k$. First, particles of color $k=0$ reproduce and move according to the rules of a branching random walk with offspring distribution Poisson- $R$, so the expected number of visits to vertex $y$ by particles of color $k=0$ is $G_{R}(1, y)$, by Proposition 2.5. This proves (39) in the case $k=0$. Second, assume that the assertion is true for color $k \geq 0$, and consider the production of particles of color $k+1$. Such particles are produced only by particles of color $k$ or color $k+1$. Call a particle a pioneer if its color is different from that of its parent, that is, if it results from a $V$-draw. Each pioneer of color $k+1$ engenders its own branching random walk of descendants with color $k+1$; the offspring distribution for this branching random walk is the Poisson- $R$ distribution. Thus, for a pioneer born at site $z \in \Gamma$, the expected number of visits to $y$ by its color- $(k+1)$ descendants is $G_{R}(z, y)$. Every particle of color $k+1$ belongs to the progeny of one and only one pioneer; consequently, the expected number of visits to $y$ by particles of color $k+1$ is

$$
\sum_{z \in \Gamma} u_{k+1}(z) G_{R}(z, y)
$$

where $u_{k+1}(z)$ is the expected number of pioneers of color $k+1$ born at site $z$ during the evolution of the branching process. But since pioneers of color $k+1$ must be children of parents of color $k$, and since for any particle the expected number of children of different color is $\varepsilon$, it follows that

$$
u_{k+1}(z)=\varepsilon v_{k}(z)
$$


Hence, Formula (39) for $k+1$ follows by the induction hypothesis.

Recall that our objective is to show that if $\sum_{x \in S_{m}} G_{R}(1, x)^{2}$ decays exponentially in $m$ then $G_{r}(1,1)<\infty$ for some $r=R+\varepsilon>R$. The branching random walk construction exhibits $G_{r}(1,1)$ as the expected total number of particle visits to the root vertex 1, and this is the sum over $k \geq 0$ of the expected number $v_{k}(1)$ of visits by particles of color $k$. Thus, to complete the proof of Proposition 7.1 it suffices, by Lemma 7.3, to show that for some $\varepsilon>0$,

$$
\sum_{k=0}^{\infty} \varepsilon^{k} \sum_{x_{1}, x_{2}, \ldots x_{k} \in \Gamma} G_{R}\left(1, x_{1}\right)\left(\prod_{i=1}^{k-1} G_{R}\left(x_{i}, x_{i+1}\right)\right) G_{R}\left(x_{k}, 1\right)<\infty .
$$

This follows directly from the next lemma.

Lemma 7.4. - Assume that Ancona's inequalities (5) hold at the spectral radius $R$ with a constant $C_{R}<\infty$. If the sum $\sum_{x \in S_{m}} G_{R}(1, x)^{2}$ decays exponentially in $m$, then there exist constants $\delta>0$ and $C, \varrho<\infty$ such that for every $k \geq 1$,

$$
\sum_{x_{1}, x_{2}, \ldots x_{k} \in \Gamma} G_{R}\left(1, x_{1}\right)\left(\prod_{i=1}^{k-1} G_{R}\left(x_{i}, x_{i+1}\right)\right)(1+\delta)^{\left|x_{k}\right|} G_{R}\left(x_{k}, 1\right) \leq C \varrho^{k} .
$$

Here $|y|=d(1, y)$ denotes the distance of $y$ from the root 1 in the word metric.

Proof. - Denote by $H_{k}(\delta)$ the left side of (40); the strategy will be to prove by induction on $k$ that for sufficiently small $\delta>0$ the ratios $H_{k+1}(\delta) / H_{k}(\delta)$ remain bounded as $k \rightarrow \infty$. Consider first the sum $H_{1}(\delta)$ : by the hypothesis that $\sum_{x \in S_{m}} G_{R}(1, x)^{2}$ decays exponentially in $m$ and the symmetry $G_{r}(x, y)=G_{r}(y, x)$ of the Green's function, for all sufficiently small $\delta>0$

$$
H_{1}(\delta):=\sum_{x \in \Gamma} G_{R}(1, x)^{2}(1+\delta)^{|x|}<\infty .
$$

Now consider the ratio $H_{k+1}(\delta) / H_{k}(\delta)$. Fix vertices $x_{1}, x_{2}, \ldots, x_{k}$, and for an arbitrary vertex $y=x_{k+1} \in \Gamma$, consider its position vis-à-vis the geodesic segment $L=L\left(1, x_{k}\right)$ from the root vertex 1 to the vertex $x_{k}$. Let $z \in L$ be the vertex on $L$ nearest $y$ (if there is more than one, choose arbitrarily). By the triangle inequality,

$$
|y| \leq|z|+d(z, y) \text {. }
$$

Because the group $\Gamma$ is word-hyperbolic, all geodesic triangles - in particular, any triangle whose sides consist of geodesic segments from $y$ to $z$, from $z$ to $x_{k}$, and from $x_{k}$ to $y$, or any triangle whose sides consist of geodesic segments from $y$ to $z$, from $z$ to 1 , and from 1 to $y$-are $\Delta$-thin, for some $\Delta<\infty$ (cf. [20] or [23]). Hence, any geodesic segment from $x_{k}$ to $y$ must pass within distance $8 \Delta$ of the vertex $z$. Therefore, by the Harnack and Ancona inequalities (15) and (5), for some constant $C_{*}=C_{R} C_{\text {Harnack }}^{32 \Delta}<\infty$ independent of $y, x_{k}$,

$$
\begin{aligned}
G_{R}(y, 1) & \leq C_{*} G_{R}(y, z) G_{R}(z, 1) \quad \text { and } \\
G_{R}\left(y, x_{k}\right) & \leq C_{*} G_{R}(y, z) G_{R}\left(z, x_{k}\right) .
\end{aligned}
$$

On the other hand, by the log-subadditivity of the Green's function,

$$
G_{R}(1, z) G_{R}\left(z, x_{k}\right) \leq C^{\prime} G_{R}\left(x_{k}, 1\right) .
$$

4 e SÉRIE - TOME $46-2013$ - No 1 
It now follows that

$$
\begin{aligned}
(1+\delta)^{|y|} G_{R}\left(x_{k}, y\right) G_{R}(y, 1) & \leq C_{*}^{2} C^{\prime}(1+\delta)^{|z|+d(z, y)} G_{R}\left(z, x_{k}\right) G_{R}(z, y) G_{R}(y, z) G_{R}(z, 1) \\
& \leq C_{*}^{2} C^{\prime}(1+\delta)^{|z|+d(z, y)} G_{R}\left(x_{k}, 1\right) G_{R}(z, y)^{2} .
\end{aligned}
$$

Denote by $\Gamma(z)$ the set of all vertices $y \in \Gamma$ such that $z$ is a closest vertex to $y$ in the geodesic segment $L$. Then for each $z \in L$,

$$
\sum_{y \in \Gamma(z)}(1+\delta)^{d(z, y)} G_{R}(z, y)^{2} \leq \sum_{y \in \Gamma}(1+\delta)^{|y|} G_{R}(1, y)^{2}=H_{1}(\delta) .
$$

Finally, because $L$ is a geodesic segment from 1 to $x_{k}$ there is precisely one vertex $z \in L$ at distance $n$ from $x_{k}$ for every integer $0 \leq n \leq\left|x_{k}\right|$, so $\sum_{z \in L}(1+\delta)^{|z|} \leq C_{\delta}(1+\delta)^{\left|x_{k}\right|}$ where $C_{\delta}=(1+\delta) / \delta$. Therefore,

$$
H_{k+1}(\delta) \leq C_{*}^{2} C^{\prime} C_{\delta} H_{1}(\delta) H_{k}(\delta)
$$

\section{Critical exponent of the Green's function at the spectral radius}

In this section we prove Theorem 1.10, using the thermodynamic formalism established in the preceding sections and the Ancona inequality (5).

\subsection{Reduction to a simple case}

Consider first the case $x=y=1$ of Theorem 1.10. The system of differential Equations (9) implies that the growth of the derivative $d G_{r}(1,1) / d r$ as $r \rightarrow R$ - is controlled by the growth of the quadratic sums $\sum_{x \in \Gamma} G_{r}(1, x)^{2}$. To show that the Green's function has a square root singularity at $r=R$, as asserted in (10), it will suffice to show that the (approximate) derivative behaves as follows as $r \rightarrow R-$ :

Proposition 8.1. - For some $0<C<\infty$,

$$
\eta(r):=\sum_{x \in \Gamma} G_{r}(1, x)^{2} \sim C / \sqrt{R-r} \quad \text { as } r \rightarrow R-.
$$

This will follow from Corollary 8.4 below. The key to the argument is that the growth of $\eta(r)$ as $r \rightarrow R$ - is related by Proposition 6.2 to that of $\operatorname{Pr}\left(2 \varphi_{r}\right)$ : in particular, Proposition 7.1 implies that $\eta(r) \rightarrow \infty$ as $r \rightarrow R-$, so the dominant contribution to the sum (41) comes from vertices $x$ at large distances from the root vertex 1. Consequently, by Equation (33),

$$
\eta(r)=\sum_{m=0}^{\infty} \sum_{x \in S_{m}} G_{r}(1, x)^{2} \sim C(R, 2) /\left(1-\exp \left\{\operatorname{Pr}\left(2 \varphi_{r}\right)\right\}\right) \quad \text { as } r \rightarrow R-.
$$

Before beginning the analysis of $\eta(r)$ near the singularity $r=R$ we will show that the relation (41) implies similar asymptotic behavior for the derivatives of all of the Green's functions $G_{r}(x, y)$.

COROLlaRY 8.2. - There exist constants $0<C_{1, y}<\infty$ such that

$$
\sum_{x \in \Gamma} G_{r}(1, x) G_{r}(x, y) \sim C_{1, y} / \sqrt{R-r} \text { as } r \rightarrow R-.
$$


Proof. - Recall the probability measures $\lambda_{r, m}$ on the spheres $S_{m}$ with densities proportional to $G_{r}(1, x)^{2}$ (see (31)), and recall that these probabilities transfer to probability measures, also denoted by $\lambda_{r, m}$, on $\Sigma^{m}\left(E_{*}\right)$, using the correspondence $S_{m} \leftrightarrow \Sigma^{m}\left(E_{*}\right)$. By Proposition 6.2, as $m \rightarrow \infty$ the measures $\lambda_{r, m}$ converge weakly to a probability measure $\lambda_{r}$, and this convergence is uniform for $r \in[1, R]$, in the sense specified by (32). These measures are related to the sum in (42) as follows:

$$
\sum_{x \in S_{m}} G_{r}(1, x) G_{r}(x, y)=\sum_{x \in S_{m}} G_{r}(1, x)^{2} \frac{G_{r}(x, y)}{G_{r}(1, x)}=\left(\sum_{x \in S_{m}} G_{r}(1, x)^{2}\right) \int \frac{G_{r}(x, y)}{G_{r}(1, x)} d \lambda_{r, m}(x) .
$$

As $x \rightarrow \xi \in \partial \Gamma$ the ratios $G_{r}(x, y) / G_{r}(1, x)$ converge to the Martin kernel $K_{r}(y, \xi)$, and the convergence is uniform, by Theorem 1.2. Hence, the weak convergence $\lambda_{r, m} \Rightarrow \lambda_{r}$ implies that

$$
\lim _{m \rightarrow \infty} \int \frac{G_{r}(x, y)}{G_{r}(1, x)} d \lambda_{r, m}(x):=C_{1, y}(r)
$$

exists, and the convergence is uniform for $r \in[1, R]$. Therefore, the corollary follows from Proposition 8.1.

\subsection{Analysis of the function $\eta(r)$ near the singularity $r=R$}

To analyze the behavior of $\eta(r)$ (or equivalently that of $\operatorname{Pr}\left(2 \varphi_{r}\right)$ ) as $r \rightarrow R-$, we use the differential Equations (9) to express the derivative of $\eta(r)$ as

$$
\frac{d \eta}{d r}=\sum_{x \in \Gamma}\left\{\sum_{y \in \Gamma} 2 r^{-1} G_{r}(1, x) G_{r}(1, y) G_{r}(y, x)\right\}-2 r^{-1} G_{r}(1, x)^{2} .
$$

(Note: The implicit interchange of $d / d r$ with an infinite sum is justified here because the Green's functions $G_{r}(u, v)$ are defined by power series with nonnegative coefficients.) For $r \approx R$, the sum $\sum_{x \in \Gamma}$ is dominated by the terms corresponding to vertices $x$ at large distances from the root 1 . Because the second term $2 r^{-1} G_{r}(1, x)^{2}$ in (43) remains bounded as $r \rightarrow R-$, it is asymptotically negligible compared to the first term $\sum_{y}$ and so we can ignore it in proving (41).

The strategy for dealing with the inner sum $\sum_{y \in \Gamma}$ in (43) will be similar to that used in the proof of Lemma 7.4 above. For each $x$, let $L=L(1, x)$ be the unique geodesic segment from the root to $x$ that corresponds to a path in the Cannon automaton, and partition the sum $\sum_{y \in \Gamma}$ according to the nearest vertex $z \in L$ :

$$
\sum_{y \in \Gamma}=\sum_{z \in L} \sum_{y \in \Gamma(z)}
$$

where $\Gamma(z)$ is the set of all vertices $y \in \Gamma$ such that $z$ is a closest vertex to $y$ in the geodesic segment $L$. (If for some $y$ there are several vertices $z_{1}, z_{2}, \ldots$ on $L$ all closest to $y$, put $y \in \Gamma\left(z_{i}\right)$ only for the vertex $z_{i}$ nearest to the root 1.) By the log-subadditivity of the Green's function and Theorem 1.3 (the Ancona inequalities) there exists a constant $C<\infty$ independent of $1 \leq r \leq R$ such that for all choices of $x \in \Gamma, z \in L(1, x)$, and $y \in \Gamma(z)$,

$$
\begin{aligned}
G_{r}(1, x) G_{r}(1, y) G_{r}(y, x) & \leq C G_{r}(1, z)^{2} G_{r}(z, x)^{2} G_{r}(z, y)^{2} \\
& \leq C G_{r}(1, x)^{2} G_{r}(z, y)^{2}
\end{aligned}
$$


consequently, for each $x \in \Gamma$,

$$
\begin{aligned}
\sum_{y \in \Gamma} G_{r}(1, x) G_{r}(1, y) G_{r}(y, x) & \leq \sum_{z \in L(1, x)} \sum_{y \in \Gamma(z)} C G_{r}(1, x)^{2} G_{r}(z, y)^{2} \\
& \leq \sum_{z \in L(1, x)} \sum_{y \in \Gamma} C G_{r}(1, x)^{2} G_{r}(z, y)^{2} \\
& =C G_{r}(1, x)^{2}(|x|+1) \eta(r) .
\end{aligned}
$$

Proposition 8.3 below asserts that there is a positive constant $\xi(r)$ such that when $C$ is replaced by $\xi(r)$ this inequality is in fact an approximate equality for "most" $x \in S_{m}$, provided $m$ is large and $r$ is near $R$. This implies that for large $m$ the contribution to the double sum in (43) with $|x|=m$ is dominated by those $x$ that are "generic" for the probability measure $\lambda_{r, m}$ on $S_{m}$ with density proportional to $G_{r}(1, x)^{2}$ (cf. Sec. 6.3).

Proposition 8.3. - For each $r \leq R$ and each $m=1,2, \ldots$ let $\lambda_{r, m}$ be the probability measure on the sphere $S_{m}$ with density proportional to $G_{r}(1, x)^{2}$. There is a continuous, positive function $\xi(r)$ of $r \in[1, R]$ such that for each $\varepsilon>0$, and uniformly for $1 \leq r<R$,

$$
\lim _{m \rightarrow \infty} \lambda_{r, m}\left\{x \in S_{m}:\left|\frac{1}{m} \sum_{y \in \Gamma} G_{r}(1, y) G_{r}(y, x) / G_{r}(1, x)-\xi(r) \eta(r)\right|>\varepsilon \eta(r)\right\}=0 .
$$

This will be deduced from Corollary 6.5 - see Section 8.3 below. Given Proposition 8.3, Proposition 8.1 and Theorem 1.10 follow easily, as we now show.

COROLlary 8.4. - There exists a positive, finite constant $C$ such that as $r \rightarrow R-$,

$$
\frac{d \eta}{d r} \sim C \eta(r)^{3} .
$$

Consequently,

$$
\eta(r)^{-2} \sim C(R-r) / 2
$$

Proof. - We have already observed that as $r$ near $R$, the dominant contribution to the sum (43) comes from vertices $x$ far from the root. Proposition 8.3 and the uniform upper bound (46) on ergodic averages imply that as $r \rightarrow R-$,

$$
\begin{aligned}
\frac{d \eta}{d r} \sim \sum_{x \in \Gamma} \sum_{y \in \Gamma} 2 r^{-1} G_{r}(1, x) G_{r}(1, y) G_{r}(y, x) & \sim 2 R^{-1} \xi(R) \eta(r) \sum_{m=1}^{\infty} m \sum_{x \in S_{m}} G_{r}(1, x)^{2} \\
& \sim C^{\prime} \eta(r) /\left(1-\exp \left\{\operatorname{Pr}\left(2 \varphi_{r}\right)\right\}\right)^{2} \\
& \sim C \eta(r)^{3}
\end{aligned}
$$

for suitable positive constants $C, C^{\prime}$. This proves (48). The relation (49) follows directly from (48). 


\subsection{Proof of Proposition 8.3}

This will be accomplished by showing that the average in (47) can be expressed approximately as an ergodic average of the form (36), to which the result of Corollary 6.5 applies. The starting point is a version of the decomposition (44), but using a continuous partition of unity instead of the characteristic functions of the sets $\Gamma(z)$, since we will need a continuous extension to the boundary of the group.

Lemma 8.5. - For $K$ large enough, we can associate to any geodesic segment $L$ in the Cayley graph of length $2 K+1$ centered at 1 a function $\gamma_{L}: \Gamma \rightarrow[0,1]$ with the following properties:

1. The function $\gamma_{L}$ extends continuously to $\bar{\Gamma}=\Gamma \cup S^{1}$.

2. For any $y \in \Gamma$, if $\pi_{L}(y)$ is the set of points on $L$ closest to $y$, then $\gamma_{L}(y)=0$ unless $\pi_{L}(y)$ is contained in the ball of radius $K / 4$ centered at 1 .

3. For any bi-infinite geodesic $L^{\prime}$ the sum of the functions $\gamma_{L}$ over all subsegments $L$ of $L^{\prime}$ of length $2 K+1$ is identically equal to 1 . More formally, for all $y \in \Gamma$,

$$
\sum_{i \in \mathbb{Z}} \gamma_{L^{\prime}(i)^{-1} L^{\prime}[i-K, i+K]}\left(L^{\prime}(i)^{-1} y\right)=1 .
$$

If $\gamma_{L}$ were defined to be the function satisfying $\gamma_{L}(y)=1$ if the closest point to $y$ on $L$ is 1 , and 0 otherwise (with ties resolved as explained in Paragraph 8.2) then the last two properties would be satisfied, but $\gamma_{L}$ would not in general extend continuously to the boundary.

Proof. - Let $K$ be even. For any geodesic segment $L_{0}$ of length $K+1$ centered around 1 , consider the set $A$ of points $y$ such that $\pi_{L_{0}}(y)$ contains a point at distance at most $K / 10$ of 1 , and the set $B$ of points $y$ such that $\pi_{L_{0}}(y)$ contains a point at distance at least $K / 5$ of 1 . By the hyperbolicity of the group $\Gamma$, if $K$ is large enough, the two sets $A, B$ are disjoint, and their closures in $\bar{\Gamma}$ are still disjoint. Therefore, there exists a continuous function $0 \leq g_{L_{0}} \leq 1$ on $\bar{\Gamma}$ equal to 1 on $\bar{A}$ and to 0 on $\bar{B}$.

If $L$ is a geodesic segment of length $2 K+1$, let $\gamma_{L}$ be equal to $g_{L[-K / 2, K / 2]}$ divided by the sum of the functions $g_{\tilde{L}}$ along every subsegment $\tilde{L}$ of $L$ of length $K+1$. Formally,

$$
\gamma_{L}(y)=g_{L[-K / 2, K / 2]}(y) / \sum g_{L(i)^{-1} L[-K / 2+i, K / 2+i]}\left(L(i)^{-1} y\right) .
$$

The sum in the denominator is $\geq 1$ on a neighborhood of the support of $g_{L[-K / 2, K / 2]}$ by construction, so the function $\gamma_{L}$ is well defined and continuous. All the properties of the lemma follow easily.

Let us now define for $r \in[1, R)$ a function $f_{r}$ on geodesic segments $L$ through 1 , as follows. Let $a$ and $b$ be the endpoints of $L$. If $d(1, a) \leq K$ or $d(1, b) \leq K$, let $f_{r}(L)=0$. Otherwise, let

$$
f_{r}(L)=\eta(r)^{-1} \sum_{y \in \Gamma} \gamma_{L[-K, K]}(y) G_{r}(a, y) G_{r}(y, b) / G_{r}(a, b) .
$$

By (50), for all $x \in \Gamma$,

$$
\eta(r) \sum_{z \in L(1, x)} f_{r}\left(z^{-1} L(1, x)\right)=\sum_{y \in \Gamma} c_{x}(y) G_{r}(1, y) G_{r}(y, x) / G_{r}(1, x),
$$

$4{ }^{\mathrm{e}}$ SÉRIE - TOME 46 - 2013 - No 1 
where the coefficient $c_{x}(y) \in[0,1]$ is equal to 1 for all points $y$ but those whose projections on $L(1, x)$ are close to 1 or $x$. By (45), the contribution of these points is bounded by $C \eta(r)$. Hence,

$$
\sum_{y \in \Gamma} G_{r}(1, y) G_{r}(y, x) / G_{r}(1, x)=\eta(r) \sum_{z \in L(1, x)} f_{r}\left(z^{-1} L(1, x)\right)+O(\eta(r)) .
$$

This quantity will be estimated thanks to Corollary 6.5 once the following lemma is established.

Lemma 8.6. - The functions $f_{r}$ are uniformly bounded and Hölder-continuous for $r \in[1, R)$, and they converge in the Hölder topology to a function $f_{R}$ as $r \rightarrow R-$.

The notion of Hölder continuity for functions of geodesic segments was defined in the discussion before Corollary 6.5.

Proof. - By definition of $\gamma_{L}$, any geodesic segment from a point $y$ with $\gamma_{L}(y)>0$ to the endpoints of the geodesic segment $L$ (or a geodesic extension of it) passes within bounded distance of 1 . Arguing as in (45), one deduces that $f_{r}$ is uniformly bounded by a constant $C$.

Consider now two geodesics $L_{1}$ and $L_{2}$ around 1 , and assume that they coincide on a neighborhood of 1 of size $n>K$. In particular, their restriction $L$ to the ball $B(1, K)$ coincide. Let $a_{1}$ and $b_{1}$ (resp. $a_{2}$ and $b_{2}$ ) be the endpoints of $L_{1}$ (resp. $L_{2}$ ). For each $y \in \Gamma$ with $\gamma_{L}(y)>0$,

$$
\frac{G_{r}\left(a_{1}, y\right) G_{r}\left(y, b_{1}\right) / G_{r}\left(a_{1}, b_{1}\right)}{G_{r}\left(a_{2}, y\right) G_{r}\left(y, b_{2}\right) / G_{r}\left(a_{2}, b_{2}\right)}=\frac{G_{r}\left(a_{1}, y\right) / G_{r}\left(a_{1}, b_{1}\right)}{G_{r}\left(a_{2}, y\right) / G_{r}\left(a_{2}, b_{1}\right)} \cdot \frac{G_{r}\left(y, b_{1}\right) / G_{r}\left(a_{2}, b_{1}\right)}{G_{r}\left(y, b_{2}\right) / G_{r}\left(a_{2}, b_{2}\right)} .
$$

Since the geodesics from $a_{1}$ or $a_{2}$ to $b_{1}$ or $y$ are fellow traveling during a time at least $n-C$ by definition (see Section 4.2), Theorem 4.6 shows that the first factor is bounded by $1+C \varrho^{n}$ for some $\varrho<1$. The second factor is bounded in the same way. Multiplying by $\eta(r)^{-1} \gamma_{L}(y)$ and summing over $y$, we obtain $f_{r}(L) \leq\left(1+C \varrho^{n}\right) f_{r}\left(L^{\prime}\right)$. Since $f_{r}$ is bounded, this yields $f_{r}(L)-f_{r}\left(L^{\prime}\right) \leq C \varrho^{n}$. Exchanging the role of $L$ and $L^{\prime}$, we get $\left|f_{r}(L)-f_{r}\left(L^{\prime}\right)\right| \leq C \varrho^{n}$. This shows that the functions $f_{r}$ are uniformly Hölder-continuous.

Next we show that the functions $f_{r}$ converge in the Hölder topology when $r \rightarrow R-$. It suffices to show that they converge pointwise, because the uniform convergence follows from the uniform Hölder bounds, and this implies convergence in the Hölder topology for any exponent strictly less than the initial exponent. Fix a geodesic segment $L$ with endpoints $a$ and $b$ : we will show that $f_{r}(L)$ converges when $r \rightarrow R-$. We have

$$
\begin{aligned}
f_{r}(L) & =\frac{1}{G_{r}(a, b)} \eta(r)^{-1} \sum_{m} \sum_{y \in S_{m}} G_{r}(1, y)^{2} \gamma_{L[-K, K]}(y) \frac{G_{r}(a, y)}{G_{r}(1, y)} \frac{G_{r}(b, y)}{G_{r}(1, y)} \\
& =\frac{1}{G_{r}(a, b)} \eta(r)^{-1} \sum_{m}\left(\sum_{y \in S_{m}} G_{r}(1, y)^{2}\right) \int F_{r}(y) d \lambda_{r, m}(y),
\end{aligned}
$$

where

$$
F_{r}(y)=\gamma_{L[-K, K]}(y) \frac{G_{r}(a, y)}{G_{r}(1, y)} \frac{G_{r}(b, y)}{G_{r}(1, y)}
$$

is a continuous function on $\Gamma$ which extends continuously to $\bar{\Gamma}$. Moreover, $F_{r}$ converges uniformly when $r \rightarrow R$ - to a limit $F_{R}$. 
By Proposition 6.2, the measures $\lambda_{r, m}$ converge as $m \rightarrow \infty$ to a measure $\lambda_{r}$ supported on $\partial \Gamma$, uniformly in $r \in[1, R]$. On the other hand, the influence of bounded $m$ in the above sum tends to 0 when $r \rightarrow R$ - since $\eta(r)=\sum_{m}\left(\sum_{y \in S_{m}} G_{r}(1, y)^{2}\right)$ tends to infinity. Therefore,

$$
\lim _{r \rightarrow R-} f_{r}(L)=\frac{1}{G_{r}(a, b)} \int_{\partial \Gamma} F_{R} d \lambda_{R}
$$

Lemma 8.6 and Corollary 6.4 imply that the convergence (47) holds with

$$
\xi(r)=\int\left(f_{r} \circ \alpha\right) d \mu_{r}^{\mathbb{Z}}
$$

That $\xi(r)$ varies continuously with $r$ for $r \leq R$ follows from the continuous dependence of the Gibbs state $\mu_{r}^{\mathbb{Z}}$ with $r$ (Theorem 6.1). Thus, to complete the proof of Proposition 8.3, it remains only to show that $\xi(R)>0$. For this it suffices to prove that there exists $C>0$ such that for all $r$ near $R$ and for $x$ with $|x|=m$, then

$$
\sum_{y \in \Gamma} G_{r}(1, y) G_{r}(y, x) / G_{r}(1, x) \geq C m \eta(r) .
$$

This sum can be partitioned by decomposing $\Gamma$ as $\bigcup \Gamma\left(z_{j}\right)$ for $z_{j} \in L(1, x)$. Hence, to prove (51) it is enough to show that each sufficiently long block of consecutive points $z_{j}$ on the geodesic segment $L=L(1, x)$ contributes at least an amount $C \eta(r)$ to the sum. This follows from the next lemma.

Lemma 8.7. - There exist $K<\infty$ and $C>0$ independent of $1 \leq r<R$ so that the following is true. For any geodesic segment $L$ of length $\geq K$ and any $K$ consecutive vertices $z_{1}, z_{2}, \ldots, z_{K}$ on $L$,

$$
\sum_{j=1}^{K} \sum_{y \in \Gamma\left(z_{j}\right)} G_{r}\left(z_{j}, y\right)^{2} \geq C \eta(r) .
$$

We will deduce this from the following statement.

Lemma 8.8. - Assume that the Cayley graph $G^{\Gamma}$ is embedded in the hyperbolic plane in such a way that the group identity $1 \in \Gamma$ is identified with the center $O$ of the Poincare disk. Then there exists $C>0$ such that for any halfplane $H$ whose boundary is a hyperbolic geodesic through $O$, and any $1 \leq r \leq R$,

$$
\sum_{x \in \Gamma: x O \in H} G_{r}(1, x)^{2} \geq C \eta(r) .
$$

Proof. - Because $\Gamma$ is co-compact, it has no parabolic elements and at least one (and therefore infinitely many) hyperbolic element. Furthermore, $\Gamma$ acts ergodically on $\partial \mathbb{D} \times \partial \mathbb{D}$, so the set consisting of all pairs $\xi^{ \pm}(x)$ of fixed points of hyperbolic elements $x$ is dense in $\partial \mathbb{D} \times \partial \mathbb{D}$. In particular, for any two antipodal points $\zeta^{+}, \zeta^{-}$on $\partial \mathbb{D}$ there are hyperbolic elements $x_{n}$ such that $x_{n}^{ \pm} O \rightarrow \zeta^{ \pm}$(in the Euclidean metric on $\mathbb{D} \cup \partial \mathbb{D}$ ) as $j \rightarrow \infty$.

Let $H$ be a halfplane whose boundary is a hyperbolic geodesic $\gamma_{H}$ through $O$, and let $\zeta^{ \pm}$ be antipodal points on $\partial \mathbb{D}$ distinct from the endpoints of $\gamma_{H}$. Precisely one of these antipodal points-say $\zeta^{+}$--will lie in the closure of $H$. Let $x_{n}$ be a sequence of hyperbolic elements such that $x_{n}^{ \pm} O \rightarrow \zeta^{ \pm}$. If $y \in \Gamma$ is such that $x_{n} y O \notin H$, for some large $n$, then by the thin triangle 
property the geodesic segment from $x_{n} O$ to $x_{n} y O$ must $\varepsilon$-shadow the geodesic segment from $x_{n} O$ to $O$, for some constant $\varepsilon<\infty$ independent of $x_{n}$ and $y$. Hence, by the translation invariance of the metric, the geodesic segment from $O$ to $y O$ must $\varepsilon$-shadow the geodesic segment from $O$ to $x_{n}^{-1} O$. Now suppose that $\zeta^{ \pm}$and $\xi^{ \pm}$are distinct antipodal pairs, neither coinciding with the endpoints of $\gamma_{H}$, and let $x_{n}, y_{n}$ be sequences of hyperbolic elements of $\Gamma$ such that $x_{n}^{ \pm} O \rightarrow \xi^{ \pm}$and $y_{n}^{ \pm} O \rightarrow \zeta^{ \pm}$. Then for all $n$ sufficiently large and all $y \in \Gamma$ either $x_{n} y O \in H$ or $y_{n} y O \in H$, because $d\left(x_{n}^{-1}, y_{n}^{-1}\right) \rightarrow \infty$.

By placing three pairs of antipodal points so that the six points are equally spaced along the circle $\partial \mathbb{D}$, we can arrange that every halfplane $H$ bounded by a hyperbolic geodesic $\gamma_{H}$ through $O$ will have at least two of the six points in the interior of the boundary $\operatorname{arc} \partial H \subset \partial \mathbb{D}$. Consequently, there exist three hyperbolic elements $h_{i} \in \Gamma$ such that for every halfplane $H$ and every $y \in \Gamma$, at least one of the six points $h_{i}^{ \pm} y O$ lies in $H$. It now follows by the trivial inequality $G_{r}(1, u v) \geq G_{r}(1, u) G_{r}(1, v)$ that

$$
\text { 6. } \sum_{x \in \Gamma: x O \in H} G_{r}(1, x)^{2} \geq\left(1 \wedge \min _{i \leq 3} G_{r}\left(1, h_{i}\right)^{2}\right) \sum_{x \in \Gamma} G_{r}(1, x)^{2} .
$$

Proof of Lemma 8.7. - Let $L$ be a geodesic segment in the Cayley graph (relative to the graph distance) of length $\geq K$, and let $z_{1}, \ldots, z_{K}$ be any $K$ consecutive vertices along $L$. By the hyperbolicity of $G^{\Gamma}$, together with the equivalence of the word and hyperbolic metrics, there is a constant $K<\infty$ such that for any choice of the geodesic segment $L$ and $K$ consecutive vertices $z_{i}$ along $L$, the region $\cup_{i=1}^{K} \Gamma\left(z_{i}\right)$ will contain a hyperbolic halfplane $H$ (actually, its intersection with $\Gamma O$ ) whose boundary is a hyperbolic geodesic that passes through exactly one vertex $g O$ located at a (graph) distance $\leq K$ from $z_{1}$. The inequality (52) now follows routinely from Lemma 8.8, by the Harnack inequality (15) and the translation invariance of the Green's function.

\section{Asymptotics of transition probabilities}

Theorem 1.11, which gives the asymptotics of transition probabilities in a co-compact Fuchsian group, is a direct consequence of the asymptotics of the Green's function given by Theorem 1.10 and of the following general statement.

Theorem 9.1. - Consider a symmetric irreducible aperiodic random walk in a countable group $\Gamma$. Let $R$ denote the radius of convergence of the Green's function $G_{z}(x, y)=\sum z^{n} p^{n}(x, y)$. Assume that there exists $\beta>0$ such that for all $x, y \in \Gamma$,

$$
G_{r}^{\prime}(x, y) \sim C_{x, y} /(R-r)^{\beta} \text { as } r \uparrow R,
$$

for some $C_{x, y}>0$. Then there exist constants $C_{x, y}^{\prime}>0$ such that

$$
p^{n}(x, y) \sim C_{x, y}^{\prime} R^{-n} n^{\beta-2} \text { as } n \rightarrow \infty .
$$

For the proof, we will rely on the following tauberian theorem of Karamata (see e.g. [6], Corollary 1.7.3 or [17], Theorem XII.5.5).

Theorem 9.2. - Let $A(z)=\sum a_{n} z^{n}$ be a power series with nonnegative coeffcients $a_{n}$ and radius of convergence 1 . Assume that, when $s$ tends to 1 along the real axis, $\sum a_{n} s^{n} \sim c /(1-s)^{\beta}$ with $\beta>0$. Then $\sum_{k=1}^{n} a_{k} \sim c n^{\beta} / \Gamma(1+\beta)$. 
Under the assumptions of Theorem 9.1, this implies asymptotics for $\sum_{k=1}^{n} k R^{k} p^{k}(x, y)$. Asymptotics of $R^{n} p^{n}(x, y)$ would readily follow if this sequence were non-increasing (see Lemma 9.5 below). This is not the case in general. However, we will show that for a symmetric, aperiodic random walk the coefficients can essentially be decomposed as $R^{n} p^{n}(x, y)=$ $q_{n}(x, y)+O\left(e^{-\delta n}\right)$ with $q_{n}(x, y)$ non-increasing and $\delta>0$; this will allow us to deduce the local limit Theorem (55).

\subsection{Spectral analysis of the transition probability operator}

The hypothesis that the random walk is symmetric implies that the Markov operator $\mathbb{P}$ of the random walk, acting on the space $\ell^{2}(\Gamma)$ by $\mathbb{P} u(x)=\sum p(x, y) u(y)$, is self-adjoint and bounded.

Theorem 9.3. - Assume that the random walk is symmetric, irreducible, and aperiodic. Then there exists $\varepsilon>0$ such that the spectrum of the Markov operator $\mathbb{P}$ is contained in the interval $\left[-R^{-1}(1+\varepsilon)^{-1}, R^{-1}\right]$. Consequently, for all $x, y \in \Gamma$ the Green's function $G_{z}(x, y)$ extends holomorphically to the doubly slit plane $\mathbb{C} \backslash((-\infty,-R(1+\varepsilon)] \cup[R, \infty))$.

Proof. - It is well known (see, for instance, [39]) that the spectrum of $\mathbb{P}$ is contained in the interval $\left[-R^{-1}, R^{-1}\right]$, where $R$ is the common radius of convergence of the Green's functions. Hence, by the spectral theorem, for any function $u \in \ell^{2}(\Gamma)$ there exists a nonnegative measure $\nu=\nu_{u}$ on $\left[-R^{-1}, R^{-1}\right]$, with total mass $\|\nu\|=\|u\|_{2}^{2}$, such that for any $n \in \mathbb{N}$

$$
\left\langle u, \mathbb{P}^{n} u\right\rangle=\int t^{n} d \nu(t),
$$

and for any complex number $z$ of modulus $|z|>R^{-1}$,

$$
R_{u}(z):=\left\langle u,(z-\mathbb{P})^{-1} u\right\rangle=\int \frac{1}{z-t} d \nu(t) .
$$

To prove the theorem it suffices to show that for some $\varepsilon>0$ the spectral measures $\nu_{u}$, where $u \in \ell^{2}(\Gamma)$, all have support contained in $\left[-R^{-1}(1+\varepsilon)^{-1}, R^{-1}\right]$.

Formula (57) exhibits the resolvent function $R_{u}(z)$ as the Stieltjes transform of the measure $\nu=\nu_{u}$. Since $\nu$ is nonnegative and has finite total mass, its Stieltjes transform $R_{u}(z)$ extends holomorphically to $\mathbb{C} \backslash\left[-R^{-1}, R^{-1}\right]$, and it satisfies the reflection identity $R_{u}(\bar{z})=\overline{R_{u}(z)}$. According to the Stieltjes inversion theorem (see, e.g., [36]), for any two real numbers $x_{1}<x_{2}$, neither of which is an atom of $\nu$,

$$
\nu\left[x_{1}, x_{2}\right]=-\lim _{y \rightarrow 0} \Im \frac{1}{\pi} \int_{x_{1}}^{x_{2}} R_{u}(x+i y) d x
$$

where $\Im$ denotes imaginary part. Suppose that $R_{u}(z)$ is analytic in a neighborhood of $\left[x_{1}, x_{2}\right]$. Since the function $R_{u}$ satisfies the reflection identity, it must be real-valued on $\left[x_{1}, x_{2}\right]$, and so it follows from the inversion Formula (58) that $\nu\left[x_{1}, x_{2}\right]=0$. Thus, to complete the proof of the theorem it suffices to show that there is some $\delta>0$ such that for every $u \in \ell^{2}(\Gamma)$, the resolvent function $R_{u}(z)$ has an analytic continuation to $\left[-R^{-1},-R^{-1}+\delta\right]$.

Observe that in the special case where $u=I_{\{1\}}$ is the indicator function of the group identity, $z^{-1} R_{u}\left(z^{-1}\right)=G_{z}(x, x)$ is the Green's function, and in the case where $u=I_{\{x, y\}}$ is the indicator of a two-point set, $z^{-1} R_{u}\left(z^{-1}\right)=2 G_{z}(x, x)+2 G_{z}(x, y)$. Hence, the Green's 
functions extend holomorphically to $\mathbb{C} \backslash((-\infty,-R] \cup[R, \infty))$. According to a theorem of Cartwright [15], for any aperiodic, symmetric random walk on a countable group the only singularity of the Green's functions $G_{z}(x, y)$ on the circle $|z|=R$ is $z=R$. In fact, his proof shows that there is an open neighborhood $U$ of $\{z:|z|=R\} \backslash\{R\}$ to which all of the functions $G_{z}(x, y)$ extend holomorphically. It follows that for $\delta>0$ sufficiently small, the resolvent function $R_{u}(z)$ extends holomorphically to $\left[-R^{-1},-R^{-1}+\delta\right]$ for all functions $u$ that are indicators of either one-point or two-point sets, and so the corresponding spectral measures attach zero mass to this interval. Since the spectral measure $\nu_{u}$ of any function $u$ with finite support can be written as a linear combination of one-point or two-point indicators, it follows that they likewise attach zero mass to the interval $\left[-R^{-1},-R^{-1}+\delta\right]$. Finally, for any $u \in \ell^{2}$ there is a sequence of finitely supported functions $u_{n}$ that converge to $u$ in $\ell^{2}$, and for any such sequence the corresponding spectral measures $\nu_{u_{n}}$ converge weakly to $\nu_{u}$. (This follows, for instance, from (56), which implies convergence of moments.) Therefore, for any $u \in \ell^{2}$ the spectral measure has support contained in $\left[-R^{-1}+\delta, R^{-1}\right]$.

Corollary 9.4. - Consider a symmetric, irreducible, aperiodic random walk on a countable group, and let $R$ be the radius of convergence of the Green's function. Then $R^{n} p^{n}(1,1)=q_{n}+O\left(e^{-\delta n}\right)$, where $q_{n}$ is nonincreasing and $\delta>0$. Furthermore, for every $x \neq 1, R^{n} p^{n}(1,1)+R^{n} p^{n}(1, x)=q_{n}(1, x)+O\left(e^{-\delta n}\right)$, where $q_{n}(1, x)$ is nonincreasing.

Proof. - Let $u$ be the indicator function of the singleton $\{1\}$, and let $\nu=\nu_{u}$ be the corresponding spectral measure. Then by (56),

$$
p^{n}(1,1)=\left\langle u, \mathbb{P}^{n} u\right\rangle=\int t^{n} d \nu(t)=\int t^{n} d \nu^{+}(t)+\int t^{n} d \nu^{-}(t),
$$

where $\nu^{+}$and $\nu^{-}$are the restrictions of $\nu$ to the positive and nonpositive reals, respectively. The sequence $q_{n}=R^{n} \int t^{n} d \nu^{+}(t)$ is clearly nonincreasing in $n$, since $\nu^{+}$is supported by the interval $\left(0, R^{-1}\right]$. By Theorem 9.3 , the support of $\nu^{-}$is contained in $\left[-R^{-1}(1+\varepsilon)^{-1}, 0\right]$ for some $\varepsilon>0$. Hence,

$$
\int t^{n} d \nu^{-}(t)=O\left(R^{-n}(1+\varepsilon)^{-n}\right)
$$

This proves the first assertion. A similar argument proves the second assertion, since

$$
2 p^{n}(1,1)+2 p^{n}(1, x)=\int t^{n} d \nu_{1, x}(t)
$$

where $\nu_{1, x}$ is the spectral measure of the indicator function of the two-point set $\{1, x\}$.

\subsection{Proof of Theorem 9.1}

Consider first the case $x=y=1$. By Corollary 9.4, we may write $R^{k} p^{k}(1,1)=q_{k}+r_{k}$ where $r_{k}$ is exponentially small and $q_{k}$ is non-increasing. Thus, to prove the asymptotic Formula (55) for $x=y=1$, it suffices to prove that

$$
q_{n} \sim C n^{\beta-2} \text { as } n \rightarrow \infty .
$$

For $s \in[0,1)$, let $r=R s$ and

$$
A(s)=r G_{r}(1,1)^{\prime}=\sum n r^{n} p^{n}(1,1)=\sum s^{n} \cdot n R^{n} p^{n}(1,1) .
$$


By hypothesis, $A(s) \sim C /(1-s)^{\beta}$ when $s \uparrow 1$. Since $R^{k} p^{k}(1,1)=q_{k}+r_{k}$ with $r_{k}$ exponentially decaying in $k$, it follows that $\sum_{k} k q_{k} s^{k} \sim C /(1-s)^{\beta}$ as $s \uparrow 1$. Therefore, Karamata's theorem gives

$$
\sum_{k=1}^{n} k q_{k} \sim C n^{\beta}
$$

The desired result now follows from the next lemma.

Lemma 9.5. - Let $q_{n}$ be a nonnegative sequence that satisfies (59) for some $\beta>0$. If $q_{n}$ is non-increasing, then as $n \rightarrow \infty$,

$$
q_{n} \sim C \beta n^{\beta-2}
$$

Proof. - Fix $\varepsilon>0$. Writing $S_{n}=\sum_{k=0}^{n-1} k q_{k}$, we have

$\varepsilon n(1-\varepsilon) n q_{n} \leq \sum_{k=(1-\varepsilon) n}^{n-1} k q_{n} \leq \sum_{k=(1-\varepsilon) n}^{n-1} k q_{k}=S_{n}-S_{(1-\varepsilon) n}=C\left(n^{\beta}-(1-\varepsilon)^{\beta} n^{\beta}+o\left(n^{\beta}\right)\right)$.

Therefore,

$$
q_{n} \leq C n^{\beta-2} \frac{1-(1-\varepsilon)^{\beta}+o(1)}{(1-\varepsilon) \varepsilon} .
$$

Letting $\varepsilon$ tend to 0 , we obtain $\lim \sup q_{n} / n^{\beta-2} \leq C \beta$. Using the interval $k \in[n,(1+\varepsilon) n]$, we control the inferior limit in the same way, and so we obtain $q_{n} \sim C \beta n^{\beta-2}$.

Finally, consider the general case $x, y \in \Gamma$. Since we have already proved the Formula (55) in the special case $x=y$, we may assume that $x \neq y$, and by homogeneity, $x=1$. By Corollary 9.4, there is a non-increasing sequence $q_{k}(1, y)$ and an exponentially decaying sequence $r_{k}(1, y)$ such that $R^{k} p^{k}(1,1)+R^{k} p^{k}(1, y)=q_{k}(1, y)+r_{k}(1, y)$. Since the Formula (55) holds for $x=y=1$, to prove it for $x=1 \neq y$ it will suffice to show that

$$
q_{k}(1, y) \sim\left(C_{1, y}^{\prime}+C_{1,1}^{\prime}\right) k^{\beta-2}
$$

for some constant $C_{1, y}^{\prime}$. (Note: By the Harnack inequality, the sequences $p^{k}(1,1)$ and $p^{k}(1, y)$ are comparable, so if this holds then $C_{1, y}^{\prime}$ must be positive.)

By Hypothesis (54),

$$
\sum_{k} s^{k} k R^{k-1}\left(p^{k}(1,1)+p^{k}(1, y)\right) \sim \frac{C_{1,1}+C_{1, y}}{(1-s)^{\beta}}
$$

as $s \uparrow 1$, and so, as in the special case considered earlier, the generating function $\sum_{k} k q_{k}(1, y) s^{k}$ satisfies the hypotheses of Karamata's theorem. Thus,

$$
\sum_{k=1}^{n} k q_{k}(1, y) \sim C n^{\beta}
$$

and so the relation (60) follows from Lemma 9.5.

$4^{\text {e }}$ SÉRIE - TOME $46-2013-$ N $^{\circ} 1$ 


\section{REFERENCES}

[1] A. Ancona, Negatively curved manifolds, elliptic operators, and the Martin boundary, Ann. of Math. 125 (1987), 495-536.

[2] A. Ancona, Positive harmonic functions and hyperbolicity, in Potential theorysurveys and problems ( Prague, 1987), Lecture Notes in Math. 1344, Springer, 1988, $1-23$.

[3] M. T. Anderson, R. Schoen, Positive harmonic functions on complete manifolds of negative curvature, Ann. of Math. 121 (1985), 429-461.

[4] K. B. Athreya, P. E. Ney, Branching processes, Grundl. der math. Wiss. 196, Springer, 1972.

[5] V. Baladi, Positive transfer operators and decay of correlations, Advanced Series in Nonlinear Dynamics 16, World Scientific Publishing Co. Inc., 2000.

[6] N. H. Bingham, C. M. Goldie, J. L. Teugels, Regular variation, Encyclopedia of Math. and its Appl. 27, Cambridge Univ. Press, 1987.

[7] S. Blachère, S. BrofFERIo, Internal diffusion limited aggregation on discrete groups having exponential growth, Probab. Theory Related Fields 137 (2007), 323-343.

[8] S. Blachère, P. Haïssinsky, P. Mathieu, Asymptotic entropy and Green speed for random walks on countable groups, Ann. Probab. 36 (2008), 1134-1152.

[9] S. Blachère, P. Haïssinsky, P. Mathieu, Harmonic measures versus quasiconformal measures for hyperbolic groups, Ann. Sci. Éc. Norm. Supér. 44 (2011), 683-721.

[10] P. Bougerol, Théorème central limite local sur certains groupes de Lie, Ann. Sci. École Norm. Sup. 14 (1981), 403-432.

[11] R. Bowen, Equilibrium states and the ergodic theory of Anosov diffeomorphisms, Lecture Notes in Math. 470, Springer, 1975.

[12] J. W. CANnON, The combinatorial structure of cocompact discrete hyperbolic groups, Geom. Dedicata 16 (1984), 123-148.

[13] J. W. Cannon, Almost convex groups, Geom. Dedicata 22 (1987), 197-210.

[14] J. W. CAnnon, The theory of negatively curved spaces and groups, in Ergodic theory, symbolic dynamics, and hyperbolic spaces (Trieste, 1989), Oxford Sci. Publ., Oxford Univ. Press, 1991, 315-369.

[15] D. I. CARTwright, Singularities of the Green function of a random walk on a discrete group, Monatsh. Math. 113 (1992), 183-188.

[16] I. Chatter Ji, K. Ruane, Some geometric groups with rapid decay, Geom. Funct. Anal. 15 (2005), 311-339.

[17] W. Feller, An introduction to probability theory and its applications. Vol. II., Second edition, John Wiley \& Sons Inc., 1971.

[18] P. Gerl, W. Woess, Local limits and harmonic functions for nonisotropic random walks on free groups, Probab. Theory Relat. Fields 71 (1986), 341-355.

[19] É. Ghys, P. De La Har Pe, Espaces métriques hyperboliques, in Sur les groupes hyperboliques d'après Mikhael Gromov (Bern, 1988), Progr. Math. 83, Birkhäuser, 1990, $27-45$. 
[20] M. Gromov, Hyperbolic groups, in Essays in group theory, Math. Sci. Res. Inst. Publ. 8, Springer, 1987, 75-263.

[21] U. HamenstäDt, Harmonic measures, Hausdorff measures and positive eigenfunctions, J. Differential Geom. 44 (1996), 1-31.

[22] M. Izumi, S. Neshveyev, R. Okayasu, The ratio set of the harmonic measure of a random walk on a hyperbolic group, Israel J. Math. 163 (2008), 285-316.

[23] I. Kapovich, N. Benakli, Boundaries of hyperbolic groups, in Combinatorial and geometric group theory (New York, 2000/ Hoboken, NJ, 2001), Contemp. Math. 296, Amer. Math. Soc., 2002, 39-93.

[24] S. KatoK, Fuchsian groups, Chicago Lectures in Mathematics, University of Chicago Press, 1992.

[25] H. Kesten, Full Banach mean values on countable groups, Math. Scand. 7 (1959), 146156.

[26] S. P. Lalley, Renewal theorems in symbolic dynamics, with applications to geodesic flows, non-Euclidean tessellations and their fractal limits, Acta Math. 163 (1989), $1-55$.

[27] S. P. Lalley, Finite range random walk on free groups and homogeneous trees, Ann. Probab. 21 (1993), 2087-2130.

[28] S. P. LALley, Random walks on regular languages and algebraic systems of generating functions, in Algebraic methods in statistics and probability (Notre Dame, IN, 2000), Contemp. Math. 287, Amer. Math. Soc., 2001, 201-230.

[29] F. Ledrappier, A renewal theorem for the distance in negative curvature, in Stochastic analysis (Ithaca, NY, 1993), Proc. Sympos. Pure Math. 57, Amer. Math. Soc., 1995, 351-360.

[30] F. LeDrappIER, Some asymptotic properties of random walks on free groups, in Topics in probability and Lie groups: boundary theory, CRM Proc. Lecture Notes 28, Amer. Math. Soc., 2001, 117-152.

[31] T. Nagnibeda, W. Woess, Random walks on trees with finitely many cone types, J. Theoret. Probab. 15 (2002), 383-422.

[32] W. Parry, M. Pollicott, Zeta functions and the periodic orbit structure of hyperbolic dynamics, Astérisque 187-188 (1990).

[33] M. Picardello, W. Woess, Examples of stable Martin boundaries of Markov chains, in Potential theory (Nagoya, 1990), de Gruyter, 1992, 261-270.

[34] C. Series, The infinite word problem and limit sets in Fuchsian groups, Ergodic Theory Dynam. Systems 1 (1981), 337-360.

[35] C. Series, Martin boundaries of random walks on Fuchsian groups, Israel J. Math. 44 (1983), 221-242.

[36] H. S. Wall, Analytic theory of continued fractions, D. Van Nostrand Company, Inc., New York, N. Y., 1948.

[37] W. Woess, A local limit theorem for random walks on certain discrete groups, in Probability measures on groups (Oberwolfach, 1981), Lecture Notes in Math. 928, Springer, 1982, 467-477.

$4^{\mathrm{e}}$ SÉRIE - TOME $46-2013-\mathrm{N}^{\circ} 1$ 
[38] W. Woess, Nearest neighbour random walks on free products of discrete groups, Boll. Un. Mat. Ital. B 5 (1986), 961-982.

[39] W. Woess, Random walks on infinite graphs and groups, Cambridge Tracts in Mathematics 138, Cambridge Univ. Press, 2000.

[40] W. Woess, Context-free pairs of groups II — cuts, tree sets, and random walks, Discrete Math. 312 (2012), 157-173.

(Manuscrit reçu le 29 juillet 2011; accepté, après révision, le 26 juin 2012.)

\author{
Sébastien GouËzel \\ Université de Rennes 1 \\ IRMAR \\ CNRS UMR 6625 \\ 35042 Rennes Cedex, France \\ E-mail: sebastien.gouezel@univ-rennes1.fr \\ Steven P. LALLEY \\ University of Chicago \\ Department of Statistics \\ 5734 University Avenue \\ Chicago IL 60637, USA \\ E-mail: lalley@galton.uchicago.edu
}

